\title{
Avaliação da Habilidade do Modelo WRF em Representar a Precipitação na Amazônia Usando Diferentes Escalas
}

\author{
Jeanne Moreira de Sousa ${ }^{1,8}$ (D), Luiz Antonio Candido ${ }^{2}$, Julio Tota da Silva ${ }^{4}$, \\ Rita Valéria Andreoli ${ }^{3}$, Mary Toshie Kayano ${ }^{5,6}$, Antonio Ocimar Manzi ${ }^{2}$, \\ Rodrigo Augusto Ferreira de Souza ${ }^{3}$, Everaldo Barreiros de Souza ${ }^{7}$, Samuel de Oliveira Vieira ${ }^{8}$ \\ ${ }^{1}$ Instituto Federal de Educação, Ciência e Tecnologia do Amazonas, \\ Presidente Figueiredo, AM, Brasil. \\ ${ }^{2}$ Instituto Nacional de Pesquisas da Amazônia, Manaus, AM, Brasil. \\ ${ }^{3}$ Universidade do Estado do Amazonas, Manaus, AM, Brasil. \\ ${ }^{4}$ Universidade Federal do Oeste do Pará, Santarém, PA, Brasil \\ ${ }^{5}$ Instituto Nacional de Pesquisas Espaciais, São José dos Campos, SP, Brasil. \\ ${ }^{6}$ Centro de Previsão de Tempo e Estudos Climáticos, São José dos Campos, SP, Brasil. \\ ${ }^{7}$ Universidade Federal do Pará, Belém, PA, Brasil. \\ ${ }^{8}$ Programa de Pós-Graduação em Clima e Ambiente, \\ Instituto Nacional de Pesquisas da Amazônia, Manaus, AM, Brasil.
}

Recebido em: 28 de Março de 2017 - Aceito em: 11 de Dezembro de 2018

\begin{abstract}
Resumo
A precipitação no norte da Amazônia dos verões e outonos austral, do período de 1988 a 1999, foi simulada utilizando o modelo regional Weather Research and Forecasting (WRF), através de uma abordagem em escalas distintas, com domínios aninhados de 45 e $15 \mathrm{~km}$. As condições iniciais e de contorno foram obtidas da Climate Forecast System Reanalysis (CFSR) do National Centers for Environmental Prediction (NCEP). A habilidade do modelo foi testada usando diferentes bases de dados observacionais de precipitação, representativas de escalas espaciais distintas. O viés do modelo mostra dependências sazonal e espacial, com viés positivo no sudoeste da Amazônia brasileira durante o verão e, no noroeste da América do Sul, durante o outono. O refinamento de escala mostrou-se necessário para reproduzir as influências de superfície nos sistemas regionais e locais que afetam a distribuição das chuvas na região. O modelo WRF, em geral, reproduz os principais padrões espaciais observados de precipitação, sem o viés seco, típico dos modelos de circulação geral da atmosfera (MCGA). Os resultados indicam que a técnica de downscaling dinâmico melhora o desempenho do modelo WRF para a previsão climática sazonal na região Amazônica.
\end{abstract}

Palavras-chave: simulação climática, WRF, América do Sul, precipitação, técnica de downscaling..

\section{Evaluation of the WRF ability to represent the precipitation in the Amazon using a different scales}

\begin{abstract}
Precipitation over the northern Amazon during the austral summer and autumn seasons of the 1988- 1999 was simulated using the Regional Weather Research and Forecasting (WRF) model, with downscaling approach with nested domains of 45 and $15 \mathrm{~km}$. The boundary and initial conditions were obtained from the Climate Forecast System Reanalysis (CFSR) of the National Centers for Environmental Prediction (NCEP). The model skill was tested using different reanalyzed precipitation datasets that represent different space scales. The bias of the model shows seasonal and spatial dependences, with positive bias in southwestern Brazilian Amazon during summer and in northwestern South America
\end{abstract}

Autor de correspondência: Jeanne Moreira de Sousa, jeanne.sousa.cliamb@gmail.com. 
during autumn. The downscaling was needed to reproduce the surface influences on the regional and local systems that affect the rainfall distribution in the region. The WRF model, in general, reproduces the main observed precipitation patterns, without the dry bias, typical of general circulation models (GCM). The results indicate that the dynamic downscaling technique improves the WRF model performance for the seasonal climate forecast in the Amazon region.

Keywords: climate simulation, WRF, South America, precipitation, downscaling technique

\section{Introdução}

Os Modelos de Circulação Geral da Atmosfera (MCGAs) têm sido ferramentas primárias utilizadas para descrever o comportamento da evolução atmosférica, associados a uma variedade de processos que ocorrem em diferentes escalas espaço-temporais. Embora o desempenho destes seja reduzido na representação de alguns processos dependentes das interações entre a atmosfera e as características da superfície (topografia, vento à superfície, microfísica de nuvens, etc.), eles representam bem os padrões de grande escala da circulação atmosférica global (Giorgi, 1990; Cavalcanti et al., 2002; Mass et al., 2002; Kinter III et al., 2009). Nas últimas décadas, os MCGAs têm tido significativos avanços em termos da representação dos processos e fenômenos essenciais para o estudo das mudanças climáticas globais (Souza e Silva, 2003; Silva et al., 2009; Leivas et al., 2011; Silveira et al., 2013) e, dentre os diversos avanços científicos está a habilidade em simular complexos sistemas físicos, constituindo uma representação matemática aproximada da realidade (Sampaio e Dias, 2014).

Os Modelos Climáticos Regionais (MCRs), também, evoluíram muito nos últimos anos e, atualmente, estão com resolução horizontal da ordem de centenas de quilômetros a metros, o que permite a representação de fenômenos de mesoescala e suas interações com a escala local em uma dada região do planeta. Estes modelos incorporam características regionais como a topografia, vegetação, solo, diferenças entre continentes e oceanos, etc., com um nível de detalhamento não usado nos MCGAs (Snyder e Sloan, 2005; Leung et al., 2006; Alves et al., 2008; Sales et al., 2015) e, por esses motivos, têm sido utilizados nos estudos das projeções climáticas regionalizadas.

A técnica denominada de "downscaling" dinâmico, que consiste em usar os resultados de MCGAs como condições de fronteira laterais nos modelos de área limitada, reúne as habilidades dos MCGAs em representar a circulação de larga escala e dos MCRs em representar os processos físicos em escala mais refinada. A solução desses processos contribui, por exemplo, para reduzir a deficiência dos MCGAs em representar a precipitação. Dessa forma, tem-se mostrado que o uso da técnica de downscaling pode trazer melhorias na representação da precipitação em relação aos MCGAs (Liu et al., 1994; Misra et al., 2003; Pesquero et al., 2010; Laprise et al., 2012; Sales et al., 2015).

A precipitação é uma das variáveis climáticas mais importantes e de difícil representação pelos MCGAs (Dai,
2006). Um dos fatores que leva a isso, como dito anteriormente, é a baixa resolução espacial dos MCGAs que não é suficiente para resolver processos de micro e meso escalas. Na Amazônia, a precipitação apresenta distribuição espaço-temporal bastante irregular (Fisch et al., 1998), e a maior parte é devido a sistemas de pequena escala que interagem com sistemas de maior escala (Cohen et al., 1989). Para Adams et al. (2009), o cisalhamento do vento, a dinâmica de pequena e grande escalas e as linhas de instabilidade exercem papel fundamental na ativação e supressão de convecção e, essa interação entre escalas, muitas vezes, não é contemplada na física dos modelos climáticos globais e, mesmo com o uso da modelagem regional, a deficiência na reprodução da precipitação sobre a Amazônia, tanto em magnitude como na distribuição espacial, ainda é muito presente.

Essa deficiência pode estar associada à representação de processos em escalas não totalmente resolvidas (Sun et al., 2005; Alves e Marengo, 2009; Silva et al., 2009; Souza et al., 2009). Por esses motivos, o objetivo deste trabalho foi o de verificar se existiria aprimoramento da habilidade do modelo para a representação da precipitação simulada, quando o espaçamento horizontal da grade dos modelos WRF, fosse progressivamente refinado incorporando diferentes escalas, simultaneamente, nas estações de verão e outono na porção tropical da América do Sul.

\section{Dados e Metodologia}

\subsection{Domínios, configuração física do modelo WRF e bases de dados}

Na Amazônia, o período chuvoso (forte atividade convectiva) ocorre de novembro a março e, o período de estiagem (fraca atividade convectiva), de maio a setembro (Fisch et al., 1998). Por esse motivo, as simulações com o modelo WRF (versão 3.1.1), descrita por Skamarock et al. (2008), foram realizadas para onze estações de verão e outono austral, abrangendo os meses de dezembro a maio, ao longo do período de 1988 a 1999. Este modelo é um dos mais avançados da atualidade e foi desenvolvido para aplicação na simulação e previsão de fenômenos atmosféricos em várias escalas, suportando o aninhamento em domínios de diferentes resoluções, permitindo a solução das equações para uma área restrita, operando assim, um downscaling gradual (Alves et al., 2003; Ferreira et al., 2008; Trail et al., 2013; Corrêa et al., 2016).

O modelo WRF foi aplicado em uma estratégia de duas grades aninhadas com resolução espacial de 45 e 
15 km de resolução espacial (Fig. 1), cujas características são apresentadas na Tabela 1. A primeira grade abrange toda a parte tropical da América do Sul, centrada na região Amazônica (domínio D01), enquanto a segunda grade inclui a porção central e leste da Amazônia (domínio D02). As condições iniciais e de contorno lateral usadas nas simulações foram obtidas da reanálise CFSR (Climate Forecast System Reanalysis)do NCEP (National Centers for Environmental Prediction) descrita em Saha et al. (2010), cuja resolução espacial é da ordem de $0.5^{\circ} \times 0.5^{\circ}$, com 64 níveis na vertical, topo da atmosfera em $0.26 \mathrm{hPa}$, e saídas a cada 6 horas. A condição de contorno sobre as áreas oceânicas foi atualizada continuamente ao longo da integração do modelo WRF utilizando as médias semanais de temperatura da superfície do mar (TSM) disponibilizados pelo CDC (Climate Diagnostic Center) do NOAA (National Oceanic and Atmospheric Administration) segundo metodologia de Reynolds e Marsico (1993).

O aninhamento entre os domínios (D01 e D02) de diferentes resoluções $(45$ e $15 \mathrm{~km}$ ) foi controlado nas suas fronteiras laterais pelo domínio D01 no sentido one-way que, além de permitir um melhor desempenho e aproveitamento do tempo computacional utilizado, os resultados da grade maior são utilizados como condição de contorno

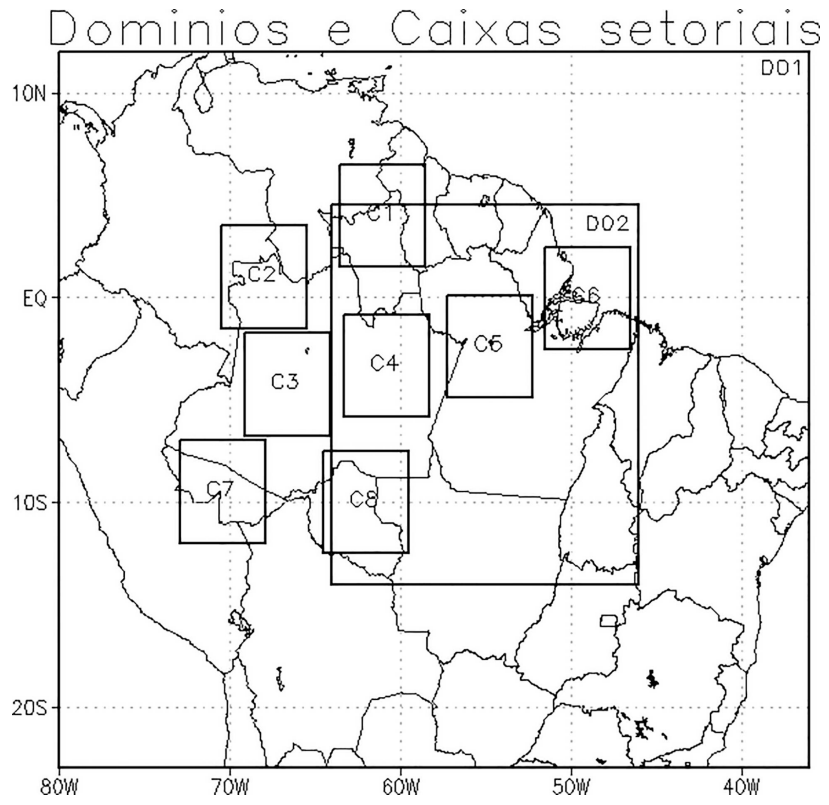

Figura 1 - Abrangência dos domínios D01 e D02, juntamente com as áreas utilizadas nas análises setoriais, chamadas Caixas setoriais, indicadas pelos códigos $\mathrm{C} 1, \mathrm{C} 2, \mathrm{C} 3, \mathrm{C} 4, \mathrm{C} 5, \mathrm{C} 6, \mathrm{C} 7$ e C8. para a grade aninhada, operando o downscaling gradualmente, fornecendo espacial e temporalmente, condições de fronteira mais refinadas para o segundo domínio (Ferreira et al., 2008; Harris e Durran, 2010; Sharma e Huang, 2012).

As integrações foram realizadas no período de 1988 a 1999 , cada uma iniciando em $1^{\circ}$ de novembro de um ano e finalizando em 31 de maio do ano seguinte, totalizando 7 meses de integração a cada par de anos (cerca de 211 dias em anos não bissextos e, 212 dias em anos bissextos). A fim de minimizar os erros devido ao ajuste dinâmico do modelo, primeiro mês (novembro) de cada rodada foi considerado como o período de spin-up e descartado das análises (Oyama et al., 2000).

O modelo WRF apresenta várias parametrizações físicas e químicas que devem ser escolhidas de acordo com o objetivo que se deseja alcançar (Ferreira et al., 2008; Litta et al., 2011; Carvalho et al., 2012; Trail et al., 2013; Oliveira, 2014). Com base na literatura existente (Carvalho et al., 2012; Dantas et al., 2013; Corrêa et al., 2016) e, na realização de testes de sensibilidade para a definição da parametrização utilizada nesta pesquisa, foram escolhidas o esquema de convecção cúmulos de Kain-Fritsch (Kain e Fritsch, 1990; 1993); a microfísica de nuvens de Lin (Lin et al., 1983), o esquema de superfície NOAH (McCaa et al., 2013) e os esquemas de radiação de ondas curtas e ondas longas RRTMG (McCaa et al., 2013).

\subsection{Dados de precipitação}

Para avaliação da habilidade do modelo em simular a precipitação foram usadas cinco bases de dados observacionais distintas: Climate Research Unit - CRU (Mitchell e Jones, 2005), Willmott - WILL (Willmot e Robeson, 1995), Global Precipitation Climatology Center - GPCC (Rudolf et al., 1994), Global Precipitation Climatology Project - GPCP (Xie et al., 2003) e Climate Prediction Center Merged Analysis of Precipitation - CMAP (Huffman et al. 2001; Xie et al., 2003). Estas bases utilizaram informações de estações de superfície distintas sobre todo o globo e, algumas também incluem informações de satélite (CMAP, GPCC e GPCP). As bases de dados denominadas por CRU, WILL e GPCC tem resolução horizontal de $0.5^{\circ}$, enquanto CMAP e GPCP tem resolução horizontal de $2.5^{\circ}$. Uma descrição detalhada destes conjuntos de dados é encontrada nas referências.

Adicionalmente, na análise dos resultados na escala de $15 \mathrm{~km}$ (D02), foi utilizada a base de dados da Rede

Tabela 1 - Características dos domínios D01 (45 km) e D02 (15 km) utilizados nas simulações com o modelo WRF.

\begin{tabular}{|c|c|c|c|c|c|c|c|}
\hline Domínio & $\Delta \mathrm{x}$ & Dimensão & Lat & Lon & Área & Topo & Níveis \\
\hline D1 (principal) & $45 \mathrm{~km}$ & $125 \times 100$ pontos de grade & $14.76^{\circ},-25.68^{\circ}$ & $82.77^{\circ}, 30.86^{\circ}$ & $25.312 .500 \mathrm{~km}^{2}$ & $50 \mathrm{hPa}$ & 38 \\
\hline D2 (aninhado) & $15 \mathrm{~km}$ & $151 \times 151$ pontos de grade & $5.70^{\circ},-15.06^{\circ}$ & $65.21^{\circ}, 44.24^{\circ}$ & $5.130 .225 \mathrm{~km}^{2}$ & $50 \mathrm{hPa}$ & 38 \\
\hline
\end{tabular}


Estadual de Previsão Climática e Hidrometeorológica do Pará (RPCH - Souza et al., 2009), que abrange grande parte do domínio D02. Na interpolação em grade, essa base regional utilizou uma maior densidade de estações meteorológicas, em comparação as bases globais, na mesma região (Fig. 2). A integração de informações de estações meteorológicas de superfície de diferentes instituições na Amazônia oriental, permitiu compor um conjunto de dados de precipitação regional com resolução horizontal de $0.27^{\circ}$. Alguns aspectos intrínsecos da distribuição pluviométrica regional, não detalhados nas bases anteriores, podem ser contemplados e avaliados na comparação entre a base RCPH e os resultados das simulações na grade de $15 \mathrm{~km}$. Além dos dados em grade, também foram utilizados as medidas de precipitação de dezoito estações meteorológicas do Instituto Nacional de Meteorologia (INMET - Tabela 2), distribuídas em sub-regiões e pertencentes as oito áreas delimitadas na Fig. 1.

\subsection{Análise da precipitação simulada nas diferentes escalas espaciais}

A habilidade do modelo WRF em simular padrões espaciais da precipitação nas estações de verão (DJF) e

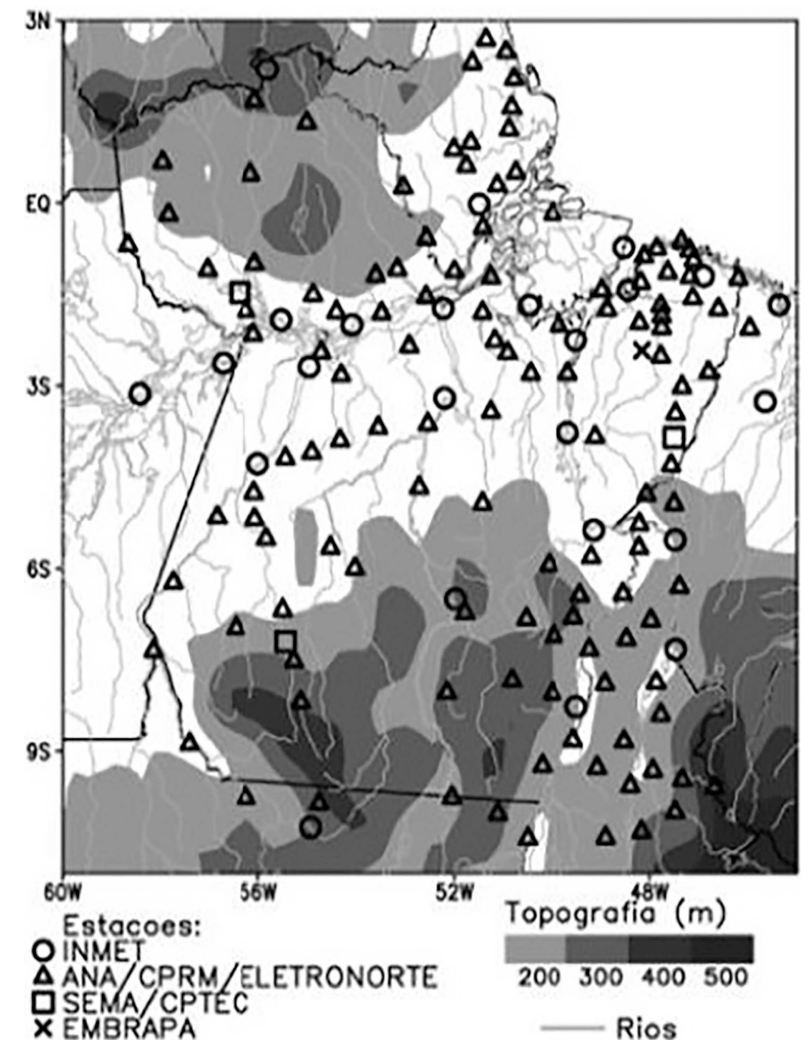

Figura 2 - Localização das estações pluviométricas (símbolos) sobre a Amazônia oriental usadas para gerar a base de dados do projeto Rede Estadual de Previsão Climática e Hidrometeorológica do Pará (RPCH), com mapa de fundo mostrando a topografia acima de $200 \mathrm{~m}$ e rede de drenagem (rios). (Fonte: Souza et al. 2009). outono (MAM) é investigada considerando as diferentes escalas horizontais de 45 e $15 \mathrm{~km}$, e também, na escala local. Para isso, parâmetros estatísticos são calculados usando os dados observados de precipitação de escala global comparados à escala de $45 \mathrm{~km}$; a base RCPH comparada à escala de $15 \mathrm{~km}$; e os dados das estações para comparação com os pontos gerados pelo "grid histiry" do WRF. A função grid history no WRF permite localizar uma estação (ou uma lista de estações), através de seus pontos de latitude e longitude e, a partir daí, gerar informações específicas para esse ponto de grade (como por exemplo, a precipitação). Esses dados de precipitação foram utilizados para avaliar a habilidade do modelo na reprodução dos totais estacionais pluviométricos em diferentes setores dos respectivos domínios.

\section{4. Índices de desempenho do modelo}

A habilidade do modelo em representar as características climatológicas da precipitação em ambos os domínios (D1 e D2) foi analisada através dos campos médios, do viés, do erro quadrático médio e da correlação de anomalias entre os dados simulados e observados, para as partes central e norte da América do Sul. Além disso, a habilidade do modelo regional em simular os padrões espaciais estacionais de precipitação foi investigada para diferentes escalas horizontais e para as estações de verão e outono.

Tabela 2 - Localização das estações de superfície do INMET, setadas no "grid histiry/tslist" do WRF.

\begin{tabular}{lccc}
\hline Caixas & Estações & Lat & Lon \\
\hline 02 & Iauaretê - AM & $00: 37$ S & $69: 12 \mathrm{~W}$ \\
& S.G. Cachoeira - AM & $00: 07 \mathrm{~S}$ & $67: 00 \mathrm{~W}$ \\
03 & B. Constant - AM & $04: 23 \mathrm{~S}$ & $70: 02 \mathrm{~W}$ \\
& Fonte Boa - AM & $02: 32 \mathrm{~S}$ & $66: 10 \mathrm{~W}$ \\
& Tefé - AM & $03: 50 \mathrm{~S}$ & $64: 42 \mathrm{~W}$ \\
04 & Barcelos - AM & $00: 58 \mathrm{~S}$ & $62: 55 \mathrm{~W}$ \\
& Coari - AM & $04: 05 \mathrm{~S}$ & $63: 08 \mathrm{~W}$ \\
& Itacoatiara - AM & $03: 08 \mathrm{~S}$ & $58: 26 \mathrm{~W}$ \\
& Manaus - AM & $03: 07 \mathrm{~S}$ & $59: 57 \mathrm{~W}$ \\
05 & Manicoré - AM & $05: 49 \mathrm{~S}$ & $61: 18 \mathrm{~W}$ \\
& Itaituba - PA & $04: 27 \mathrm{~S}$ & $55: 29 \mathrm{~W}$ \\
& Parintins - AM & $02: 38 \mathrm{~S}$ & $56: 44 \mathrm{~W}$ \\
06 & Santarém - PA & $02: 25 \mathrm{~S}$ & $54: 42 \mathrm{~W}$ \\
& Belém - PA & $01: 27 \mathrm{~S}$ & $48: 28 \mathrm{~W}$ \\
& Macapá - AP & $00: 02 \mathrm{~S}$ & $51: 03 \mathrm{~W}$ \\
& Soure - PA & $00: 40 \mathrm{~S}$ & $48: 33 \mathrm{~W}$ \\
& Cruzeiro do Sul & $07: 63 \mathrm{~S}$ & $72: 67 \mathrm{~W}$ \\
& Rio Branco & $09: 97 \mathrm{~S}$ & $67: 80 \mathrm{~W}$ \\
\hline \multirow{4}{*}{07} & & &
\end{tabular}




\section{Resultados}

\subsection{Características climatológicas da precipitação}

\subsubsection{Análise espacial na escala de $45 \mathrm{~km}$}

No trimestre DJF, o modelo WRF na escala de $45 \mathrm{~km}$, representa a área de intensa precipitação e o seu posicionamento sobre o oceano Atlântico Tropical Norte, associado à configuração da ZCIT, localizada em torno de $3^{\circ} \mathrm{N}$ (Fig. 3a), compatível com o que se verifica nas bases de dados observados CMAP e GPCP (Figs. 3e-3f), porém apresentando precipitação média superior ao observado.

Sobre o continente, o modelo simula a banda de precipitação intensa que se estende desde a parte oeste da Amazônia até o sudeste do Brasil, orientada de NW-SE, associada à ZCAS (Kodama, 1992), com precipitação média (de 300 a $600 \mathrm{~mm}$ ), superior ao observado em todas as bases de dados (que varia de 200 a $400 \mathrm{~mm}$ ). Embora, também se perceba uma diferenciação na região de ocorrência dessa banda de precipitação entre os dados observados, em que os dados CRU, WILL e GPCC apresentam áreas de valores maiores (Figs. 3b-3d) quando comparada aos dados CMAP e GPCP (Figs. 3e-3f). O WRF também captura as áreas de máxima precipitação média observadas ao longo do Amapá e noroeste do Pará (Fig. 3a), assim como as áreas menos chuvosas nessa época do ano entre Roraima, Venezuela e Colômbia, e grande parte do semiárido brasileiro, onde os valores de precipitação média são abaixo de $100 \mathrm{~mm}$. Valores máximos de precipitação são sistematicamente apresentados pelo modelo WRF na região sobre a costa oeste da $\mathrm{AS}$, pela influência da Cordilheira dos Andes nos mecanismos de convecção por levantamento (Fig. 3a), percebidos mais claramente nos dados CRU, WILL e GPCC (Figs. 3b-3d). Essa influência se prolonga ao longo do continente onde o WRF mostra bandas de precipitação média mais intensas e paralelas entre si avançando desde a Bolívia até a porção central da Amazônia (Fig. 3a).

Nitidamente, o modelo WRF representa precipitação média de magnitude maior que as observações nas áreas chuvosas e menores nas áreas que recebem pouca precipitação (por exemplo, o oeste do Nordeste). As bases de dados CRU, WILL e GPCC apresentam magnitudes um pouco maiores, comparadas as bases CMAP e GPCP, dependo da região (melhor verificada na análise sub-

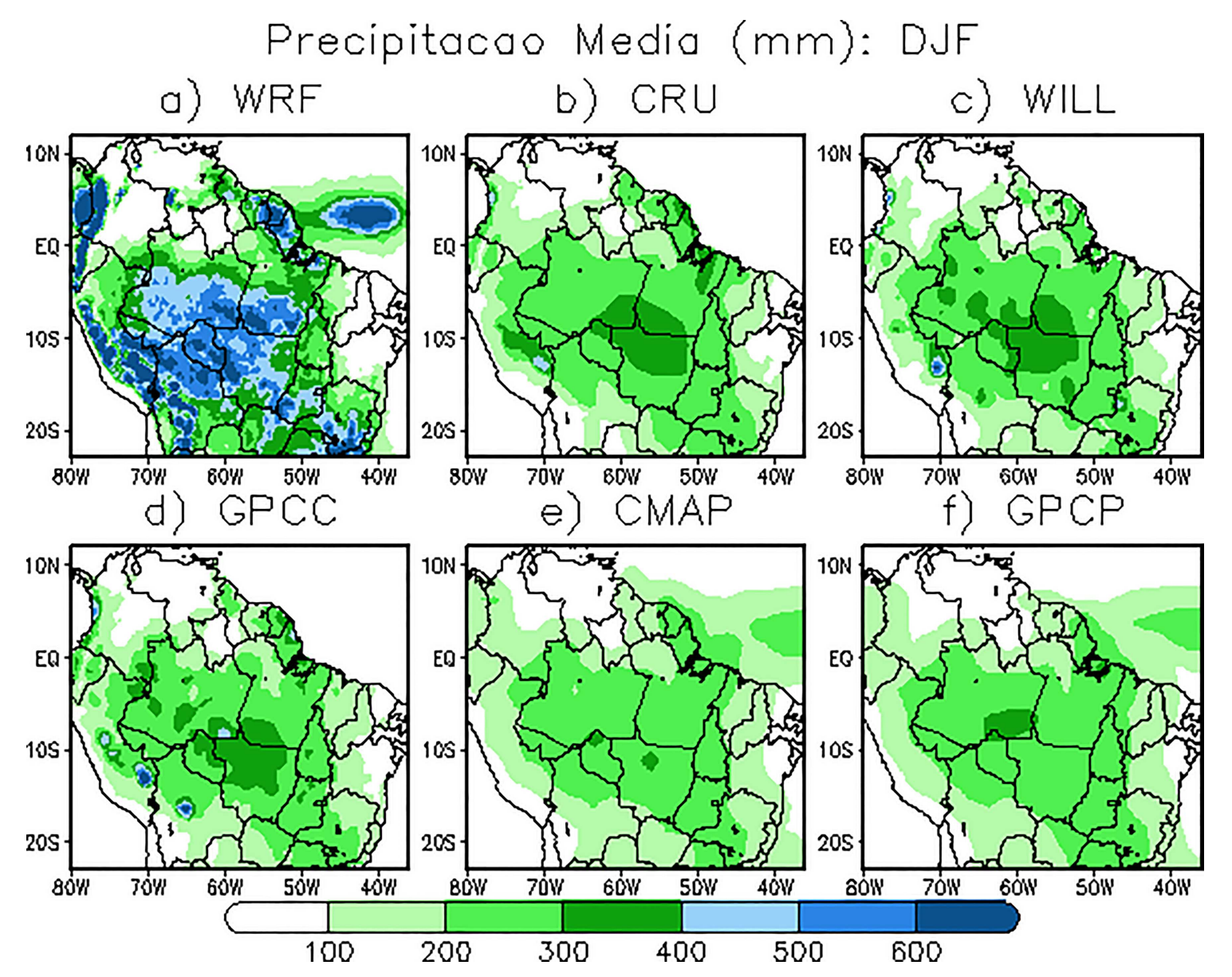

Figura 3 - Climatologias de precipitação (mm) simulada pelo modelo (a) WRF, na grade de $45 \mathrm{~km}$, comparadas às bases observacionais (b) CRU, (c) WILL, (d) GPCC, (e) CMAP e (f) GPCP, para o trimestre DJF de 1988 a 1999. 
regional). Por outro lado, os dados do CMAP e GPCP permitem a validação do WRF quanto ao posicionamento da ZCIT sobre o continente e oceano Atlântico, cujo deslocamento mais para o sul é condizente com a climatologia do período (Ribeiro et al., 1996; Souza e Ambrizzi, 2004; Reboita et al., 2010). A ZCIT mais próxima do continente e a configuração da banda de precipitação associada à ZCAS é mais ampliada no WRF, com distribuição da precipitação em áreas maiores sobre a Amazônia, mantendo maiores magnitudes de precipitação frente às observações.

No trimestre MAM (Fig. 4), a banda de precipitação se apresenta zonalmente orientada estendendo-se do extremo oeste da Amazônia até o Atlântico Tropical junto a costa da AS, nas bases observacionais (Figs. 4b-4f). Duas áreas com maiores precipitações médias são observadas sobre o continente: a primeira na costa norte da AS (nos limites do Amapá, Guiana, litoral do Pará e do Maranhão), associados à proximidade da ZCIT e aos sistemas de linhas de instabilidade (LIS - Cohen et al., 1989) típicos dessa época do ano e, a outra área de grande precipitação média nesta estação ocorre na região formada pela divisa do Brasil, Peru e Colômbia, e que se mostra aparente apenas nos dados WILL e GPCC (Figs. 4c-4b). O WRF representa essa banda de precipitação e as duas áreas de grande magnitude na costa e a oeste do continente (Fig. 4a), porém configurando uma área adicional de precipitação média mais elevada na porção central da Amazônia e, que é mais aparente na base GPCC (Fig. 4d). Nesta época do ano, a ZCIT é mais ampla e se posiciona próximo à costa norte da $\mathrm{AS}$, definindo a estação chuvosa no norte do Nordeste Brasileiro (NEB), conforme os dados CMAP e GPCP (Figs. 4e-4f). O WRF representa a posição da ZCIT, porém com banda mais larga que nas observações e maior magnitude de precipitação média (Fig. 4a).

A sistemática representação de precipitação média com valores acima da climatologia observada será discutida na seção de estudo do viés do modelo. Na porção central e no sudeste do Brasil, assim como no norte e litoral sul do nordeste brasileiro, o WRF captura os padrões climatológicos da precipitação média, espacialmente e em termos de magnitude, muito próximos aos campos observados CRU, WILL, GPCC, CMAP e GPCP (Figs. 4a-4f).

\section{Precipitacao Media (mm): MAM}

a) WRF

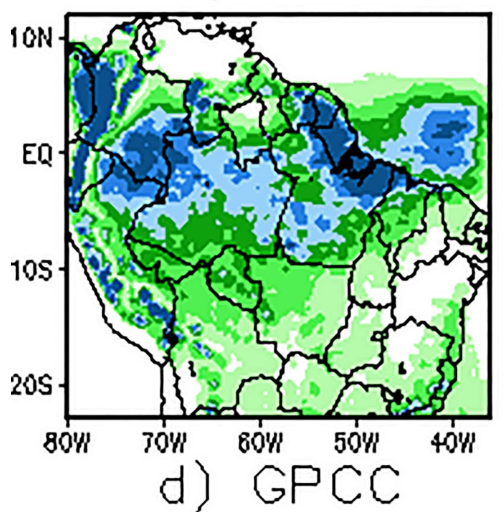

d) GPCC

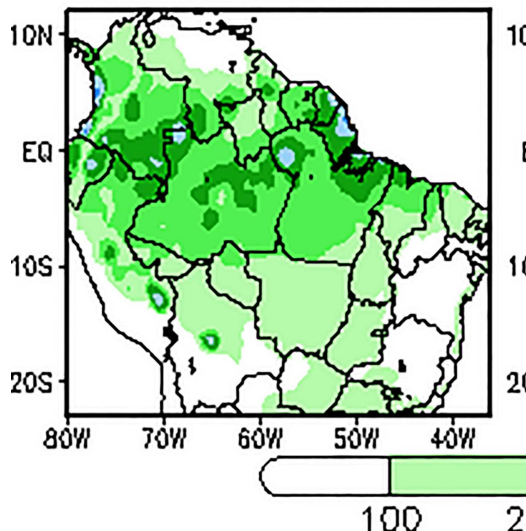

b) CRU

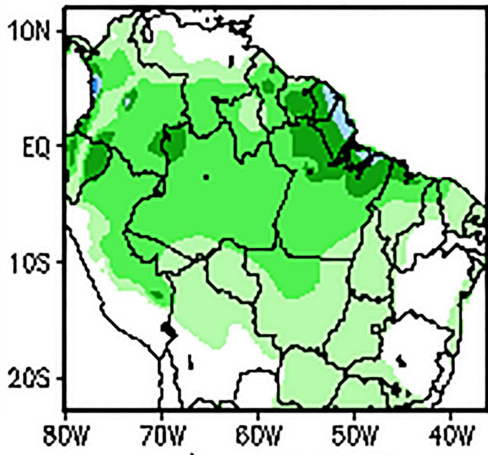

e) CMAP
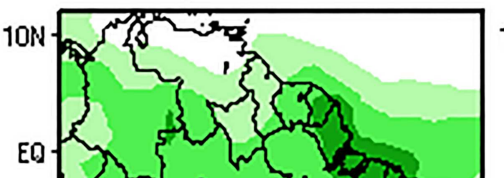

c) WILL

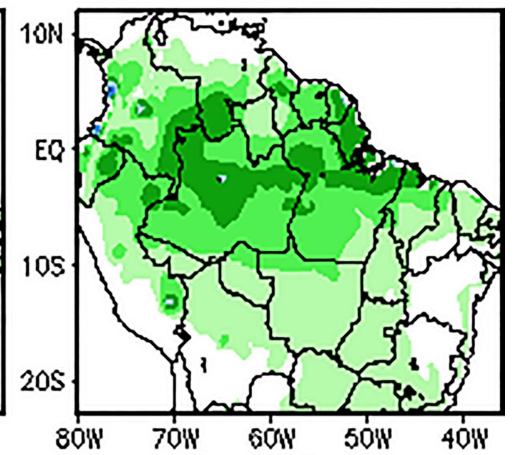

f) GPCP

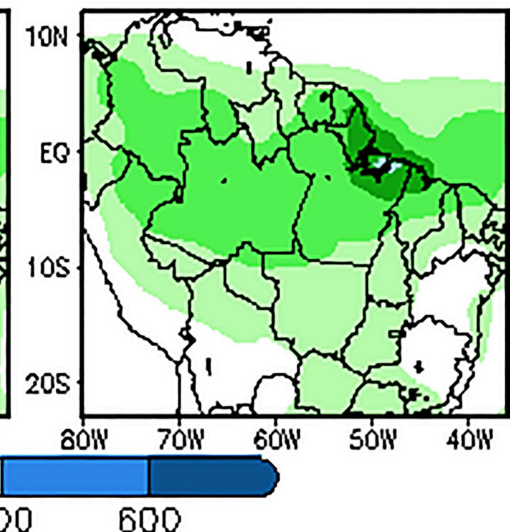

Figura 4 - Climatologias de precipitação $(\mathrm{mm})$ simulada pelo modelo (a) WRF, na grade de $45 \mathrm{~km}$, comparada às bases observacionais (b) CRU, (c) WILL, (d) GPCC, (e) CMAP e (f) GPCP, para o trimestre MAM de 1988 a 1999. 


\subsubsection{Análise espacial na escala de $15 \mathrm{~km}$}

Mapas de precipitação média para as estações DJF e MAM simulados na resolução mais fina $(15 \mathrm{~km})$ são apresentados em comparação com os dados observados Figs. 5-6). Alguns aspectos regionais intrínsecos da alta variabilidade espacial da pluviometria tropical são reproduzidos pelo WRF. As observações obtidas da base RCPH confirmam um acentuado aumento de precipitação a partir de $52^{\circ}$ de longitude oeste (Fig. 5b). Essa zona seca transversal, exatamente a leste desta longitude, avança na direção noroeste do Pará e, é claramente mostrada nas observações e representada pelo WRF (Fig. 5a). Na porção sudoeste do Pará se observa uma zona mais úmida, com precipitação média na estação superior à $300 \mathrm{~mm}$.

A distribuição topográfica para a grade fina é mostrada na Fig. 2. Nessa escala, existem algumas áreas importantes de maior elevação, como a cadeia montanhosa Tumucumaque, que abrange desde o noroeste do Pará, o oeste do Amapá e o sul da Guiana Francesa e do Suriname. Além de parte do planalto central brasileiro no sul do Pará e, as chapadas das Mangabeiras e da Mesa no sul do Maranhão. Nestas áreas, o modelo WRF apresenta forte relação com o relevo simulando chuvas médias mais intensas (acima de $400 \mathrm{~mm}$ ) em ambas estações (DJF e MAM), ocorrendo exatamente nas áreas dos altos relevos (Figs. 5a e 5c). Apesar da maior densidade da rede de monitoramento utilizada na base de dados RCPH, estes padrões espaciais da precipitação média não são capturados pelas observações (Figs. 5b e 5d) pois são áreas remotas e sem pluviômetros.

$\mathrm{Na}$ região costeira, em ambas as estações, a distribuição de precipitação média apresenta valores mais elevados no WRF (Figs. 5a e 5c) que nas observações (Figs. 5b e 5d), cuja influência não se deu apenas pela presença da ZCIT, mas também pelo desenvolvimento das circulações locais associadas ao aquecimento diferencial continente-oceano, como a brisa do mar e também o variado relevo local que, na escala mais fina são melhor capturadas. A maior precipitação nestas estações é causada pela proximidade da ZCIT, que está localizada sobre essas posições de baixa latitude. Assim, os ventos e a umidade convergem sobre a região causando alta frequência de células convectivas. Na comparação com as observações se verifica que o WRF simula altos valores de precipitação média ao longo de todo litoral norte do domínio, incluindo a área do oceano Atlântico.

Na porção central da grade de $15 \mathrm{~km}$, a topografia é dominada por terras baixas que começam na Ilha de Marajó na costa atlântica e seguem até o vale do rio Amazonas (Fig. 2). Nessa área, o WRF representa precipitação média mais baixa em MAM acompanhando o vale do rio (Fig. 5c). A base de dados RCPH descreve a variabilidade de mesoescala nesta região mais do que as outras bases de escala global, mas as características muito localizadas não são resolvidas. Essas características não são representadas pela base de dados RCPH, mas são explicadas em vários estudos pela influência da brisa de rio, neste caso do rio Amazonas, onde a diferença de rugosidade entre as superfícies do rio e o continente circundante faz com que o vento da camada limite seja canalizado ao longo do percurso do rio, resultando em subsidência induzida por brisa, e, portanto, favorecendo menor precipitação (Oliveira e Fitzjarrald, 1993; Fitzjarrald et al., 2008).

Os modelos de área limitada mostram um variado grau de habilidade nas simulações da condição do clima presente, que depende de suas características dinâmicas e físicas, assim como das condições de fronteira laterais, conforme os resultados de vários trabalhos (Alves e Marengo 2009; Pesquero et al., 2009; Rocha et al., 2009; Silvestri et al., 2009; Chou et al., 2012; Solman et al., 2013). Os nossos resultados mostram que o WRF capturou com maior fidelidade os padrões observados da precipitação, em especial sobre a Amazônia, muito diferente dos trabalhos anteriores. Esse melhor desempenho não significa que o WRF é perfeito, mas que, como em todos os estudos, as simulações regionais apresentam erros sistemáticos que estão relacionados à sua configuração física e as condições de fronteira lateral.

No nosso caso, o excesso de precipitação foi associado ao esquema de convecção profunda Kain-Fritsch usado nas simulações. Esse esquema define a escala de tempo de uma nuvem com base em uma estimativa do período de tempo advectivo para nuvens convectivas profundas, dentro de uma célula de grade, conforme estudos de ciclo de vida das nuvens (Gilliland e Rowe, 2007; Sharma e Huang, 2012). Essa escala de tempo, frequentemente resulta em ajustes termodinâmicos relativamente rápidos e altas taxas de precipitação. No caso das condições laterais, a maior qualidade dos dados de reanalise em alta resolução espacial, como o CFSR, também permitiram que erros sistemáticos úmidos sejam favorecidos, como ocorrido nestas simulações.

\subsection{Análise sub-regional da climatologia de precipitação}

A avaliação da distribuição da precipitação simulada foi também feita em setores da região Amazônica para contemplar uma análise em escala sub-regional. A Fig. 6 mostra a precipitação sazonal simulada e observada, mediadas em oito setores: Caixas 01-08. Nas Caixas 05 e 06, que são setores contidos em ambos domínios, as precipitações simuladas nas grades de $15 \mathrm{~km}$ e de $45 \mathrm{~km}$ e as observadas nas bases CRU, CMAP, GPCC, WILL, GPCP e RPCH são mostrados juntamente com o propósito de examinar as diferenças das escalas apropriadamente.

$\mathrm{Na}$ escala de $45 \mathrm{~km}$, o modelo captura a evolução sazonal da precipitação de DJF a MAM, que se caracteriza por sua intensificação associada ao avanço da estação chuvosa da Amazônia, nas sub-regiões do noroeste (Caixa 
02), norte (Caixa 01), nordeste (Caixa 06), leste (Caixa. 05) e central (Caixa 04), e seu enfraquecimento nas subregiões sul (Caixa 07) e sudoeste (Caixa 08), todas apresentadas na Fig. 6. Essa evolução das chuvas é consistente com a observação, porém, com erros sistemáticos distintos decorrentes das influências combinadas de sistemas de larga escala, mesoescala e local (Fisch et al., 1998).

$\mathrm{Na}$ Caixa 01, as precipitações simuladas na escala de $45 \mathrm{~km}$ e as observadas têm magnitudes próximas, com os menores valores em DJF e maiores em MAM, o que a) WRF $15 \mathrm{~km}$

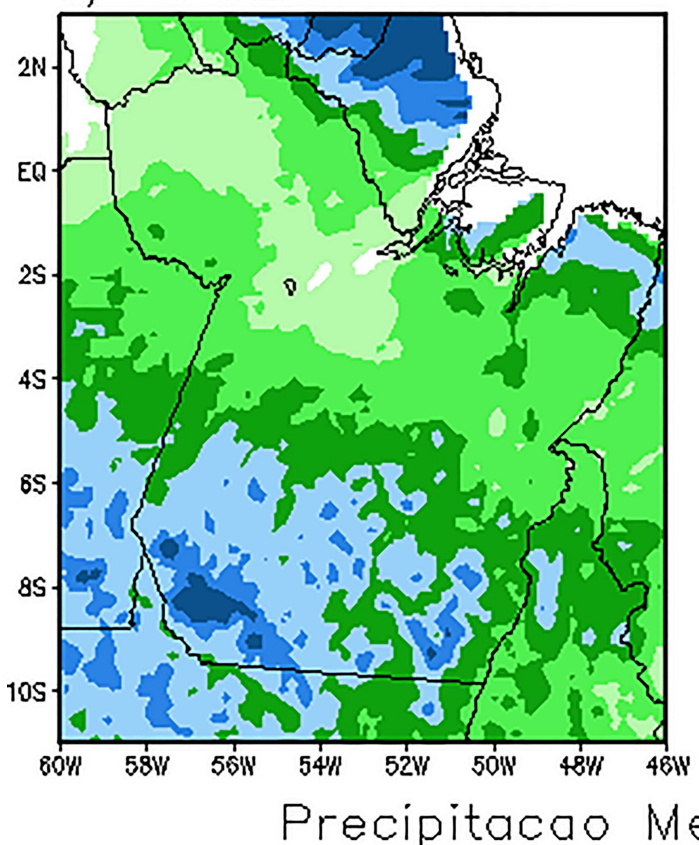

c) WRF $15 \mathrm{~km}$

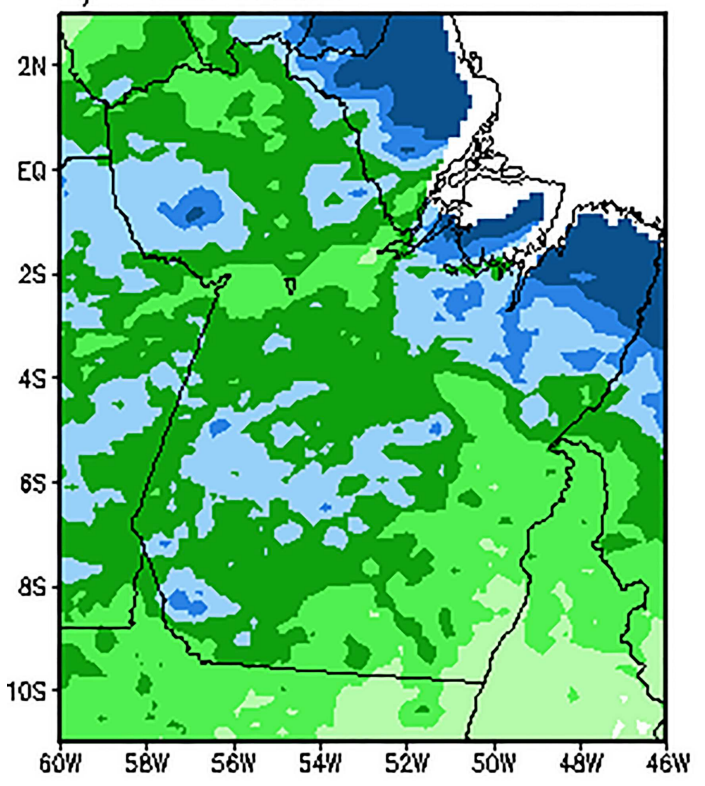

b) $\mathrm{RPCH}$

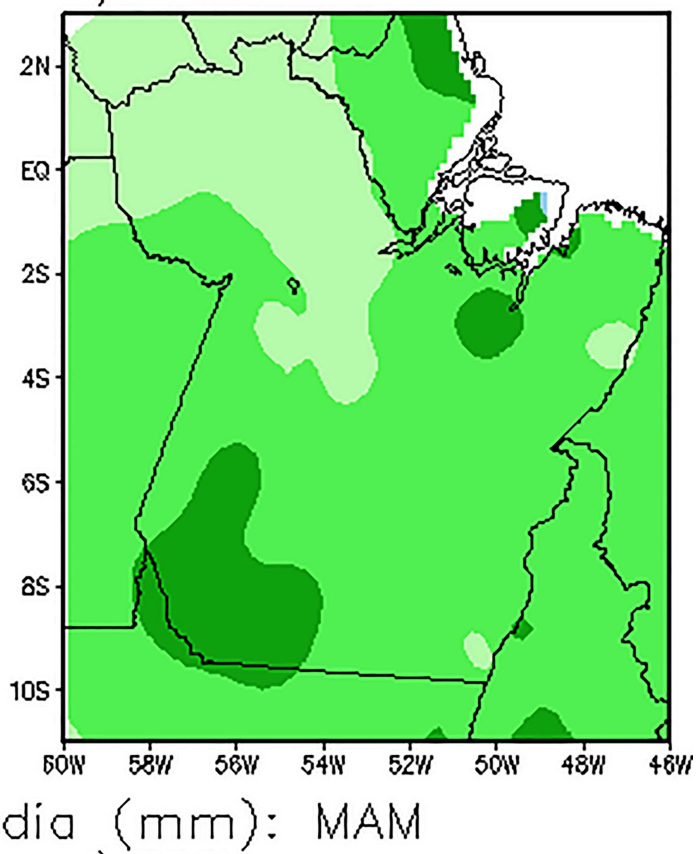

d) $\mathrm{RPCH}$

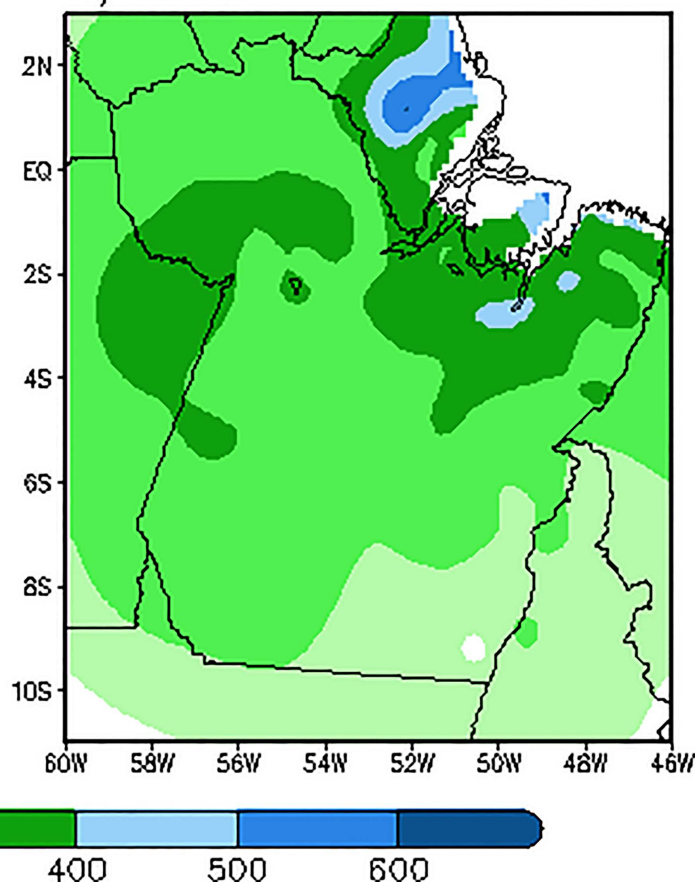

Figura 5 - Climatologia de precipitação $(\mathrm{mm})$ simulada pelo modelo (a) WRF, na grade de $15 \mathrm{~km}$, comparada à base observacional (b) RPCH, para o trimestre DJF de 1988 a 1999. Analogamente, temos a climatologia de precipitação (mm) do trimestre MAM, simulada pelo (c) WRF, comparada à base (d) $\mathrm{RPCH}$, para o mesmo período. 
reflete a variabilidade sazonal típica do extremo norte da Amazônia brasileira, que apresenta um regime de chuvas diferenciado das demais áreas (Reboita et al., 2010). Nas Caixas 02 e 03, localizadas no extremo oeste da Amazônia brasileira, as precipitações observadas têm pequenas diferenças entre as bases de dados em ambos os trimestres. Os erros inerentes aos conjuntos de dados de precipitação decorrem das diferentes resoluções espaciais, da cobertura

\section{Precipitação média nas Caixas setoriais}

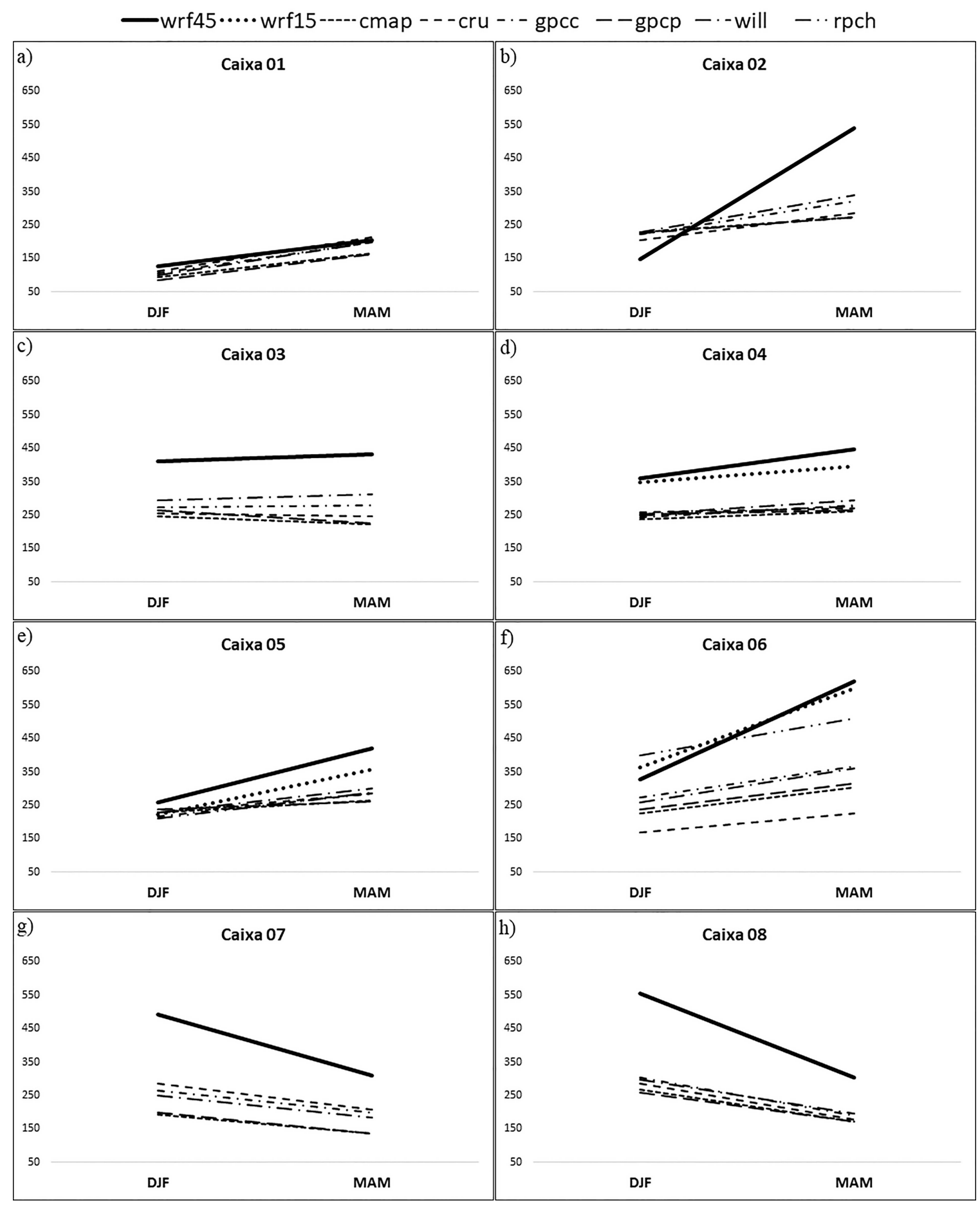

Figura 6 - Precipitação sazonal média (mm) simulada e observada, durante os trimestres DJF e MAM, nas (a) Caixa 01, (b) Caixa 02, (c) Caixa 03, (d) Caixa 04, (e) Caixa 05, (f) Caixa 06, (g) Caixa 07 e (h) Caixa 08, sub-regiões dos domínios D01 e D02. 
temporal e dos métodos utilizados em sua geração (Negron Juárez et al., 2009). Na simulação, os resultados mostram que, na Caixa 02 ocorre uma leve subestimação da precipitação em DJF e, forte superestimação em MAM. Esse erro sistemático é associado ao aumento da convergência do fluxo de umidade na região da Caixa $02 \mathrm{em}$ MAM, em que, a maior disponibilidade de umidade reforça o potencial de energia disponível à convecção. Como resultado, os esquemas de convecção e microfísica de nuvens, usadas no WRF, respondem com mais precipitação e, essa característica é verificada também nos demais setores da Amazônia, conforme mostrado a seguir.

$\mathrm{Na}$ Caixa 03, a precipitação simulada é superestimada nas duas estações, mas com a variação sazonal de chuva similar à observada. Na Caixa 04, localizada na Amazônia central, a simulação em $45 \mathrm{~km}$ superestima as precipitações para ambos os trimestres, com os maiores valores em MAM. Embora as precipitações simuladas na escala de $15 \mathrm{~km}$ ainda sejam superestimadas, são menores do que as simuladas na escala de $45 \mathrm{~km}$. Ressalta-se que esta comparação se refere às bases de dados globais de precipitação e, mesmo assim, indica uma redução do erro sistemático nessa área associado ao refinamento de escala. Na Caixa 05, localizada no leste da Amazônia oriental, as precipitações simuladas com ambas as grades (15 e $45 \mathrm{~km}$ ) e as observadas, têm pequenas diferenças em DJF, e maiores diferenças em MAM. Nesta caixa, para a escala de $15 \mathrm{~km}$, os erros são menores do que os da Caixa 04. A Caixa 06, localizada no noroeste da Amazônia, embora inclua porções de continente e oceano, apenas a parte continental deste setor é considerada na comparação com os dados observados.

Nesta área em ambas as estações (DJF e MAM), as precipitações simuladas nas duas escalas (45 e $15 \mathrm{~km}$ ) têm diferenças muito pequenas, mas as precipitações observadas mostram diferenças entre as bases de dados, o que implica em erros sistemáticos distintos dos simulados. Essas diferenças na precipitação observada é resultado da composição dos dados utilizados, que não refletem inteiramente a condição da forte interação continente-oceano, marcado por sistemas meteorológicos de escala bem inferior. Nas Caixas 07 e 08, no setor sudoeste da Amazônia, as precipitações simuladas são superestimadas, mas mostram uma evolução sazonal semelhante à observada, com maiores valores em DJF e menores em MAM.

De maneira geral, o modelo captura as localizações espaciais dos máximos e mínimos climatológicos da precipitação representados pelas Caixas de 01 a 08 , assim como sua evolução sazonal do verão para o outono, contudo, existem diferenças nas magnitudes em relação aos dados observados e, estas diferenças dependem da localização. Os erros sistemáticos foram mais elevados quando se utilizou os dados da reanálise CFSR como condições de fronteira lateral, mas ainda, o modelo teve habilidade para representar os padrões climatológicos estacionais diferenciados e inerentes a cada área.

\subsection{Avaliação dos índices de desempenho}

Os erros sistemáticos e a habilidade do modelo são avaliados através do viés, raiz quadrada do erro médio (REQM) e correlação espacial entre os campos observados e simulados de precipitação nas escalas de 45 e $15 \mathrm{~km}$ considerando separadamente as estações de DJF e MAM.

\subsubsection{Viés e correlação na escala de $45 \mathrm{~km}$}

As Figs. 7-8 apresentam os mapas do viés e, as Figs. 9-10 os mapas da correlação, entre precipitações simuladas e observadas na escala de $45 \mathrm{~km}$, nas estações de DJF e MAM. Para cada estação, os mapas do viés são muito semelhantes entre si, considerando todos os conjuntos de dados analisados, com valores positivos sobre áreas tipicamente úmidas nestas estações do ano, e valores negativos em áreas como menor conteúdo de umidade. Para ambos os trimestres (DJF e MAM), ocorre predominância de viés positivo, ou úmido, em grande parte da Amazônia, com pequenas diferenças associadas às bases de dados (Figs. 7-8). Grande viés positivo é notado no setor sudoeste de D01 (centro do Peru, Bolívia e centro-sul do Brasil) em DJF (Fig. 7), e em seu setor noroeste (norte do Peru, grande parte da Colômbia e norte do estado do Amazonas), assim como na costa leste do Pará e sobre o Amapá, em MAM (Fig. 8). Pequenos vieses negativos ocorrem em áreas do noroeste, norte e nordeste da AS, incluindo o leste da região central do Brasil e norte de Minas Gerais durante DJF (Fig. 7). Outras áreas apresentam vieses negativos (menores que $-50 \mathrm{~mm}$ ) incluindo o Equador, grande parte da Colômbia, sul da Venezuela, Guiana e estendendo-se também sobre o norte do Amazonas e Roraima e, semiárido do NEB (Fig. 7). A área de viés negativo em MAM é restrita ao setor norte da Venezuela e Roraima, onde ocorrem pequenas áreas com viés negativo (inferiores à $-50 \mathrm{~mm}$ ). Viés negativo, variando de zero a $-50 \mathrm{~mm}$, ocorre também no Brasil central e NEB, sendo mais expressivo nesta última área (Fig. 8).

Consistentemente, as maiores correlações significativas durante DJF (valores superiores a 0.7) são encontrados na área com o menor viés nesta estação, localizadas principalmente sobre o norte da AS e ao longo da área de influência da ZCIT no oceano Atlântico, para os conjuntos de dados CMAP (Fig. 9d) e GPCP (Fig. 9e). Por outro lado, as maiores correlações significativas durante MAM (valores superiores a 0.7) são encontrados no NEB e Amazônia oriental para as bases de dados CRU (Fig. 10a), GPCC (Fig. 10b) e WILL (Fig. 10c), e sobre as áreas da Amazônia central e ao longo da área de influência da ZCIT no oceano Atlântico, para os conjuntos de dados CMAP (Fig. 10d) e GPCP (Fig. 10e). Nesta estação (DJF), as maiores correlações são mais dispensas no espaço e entre as bases de dados (Figs. 9a-9e) onde, por exemplo, 


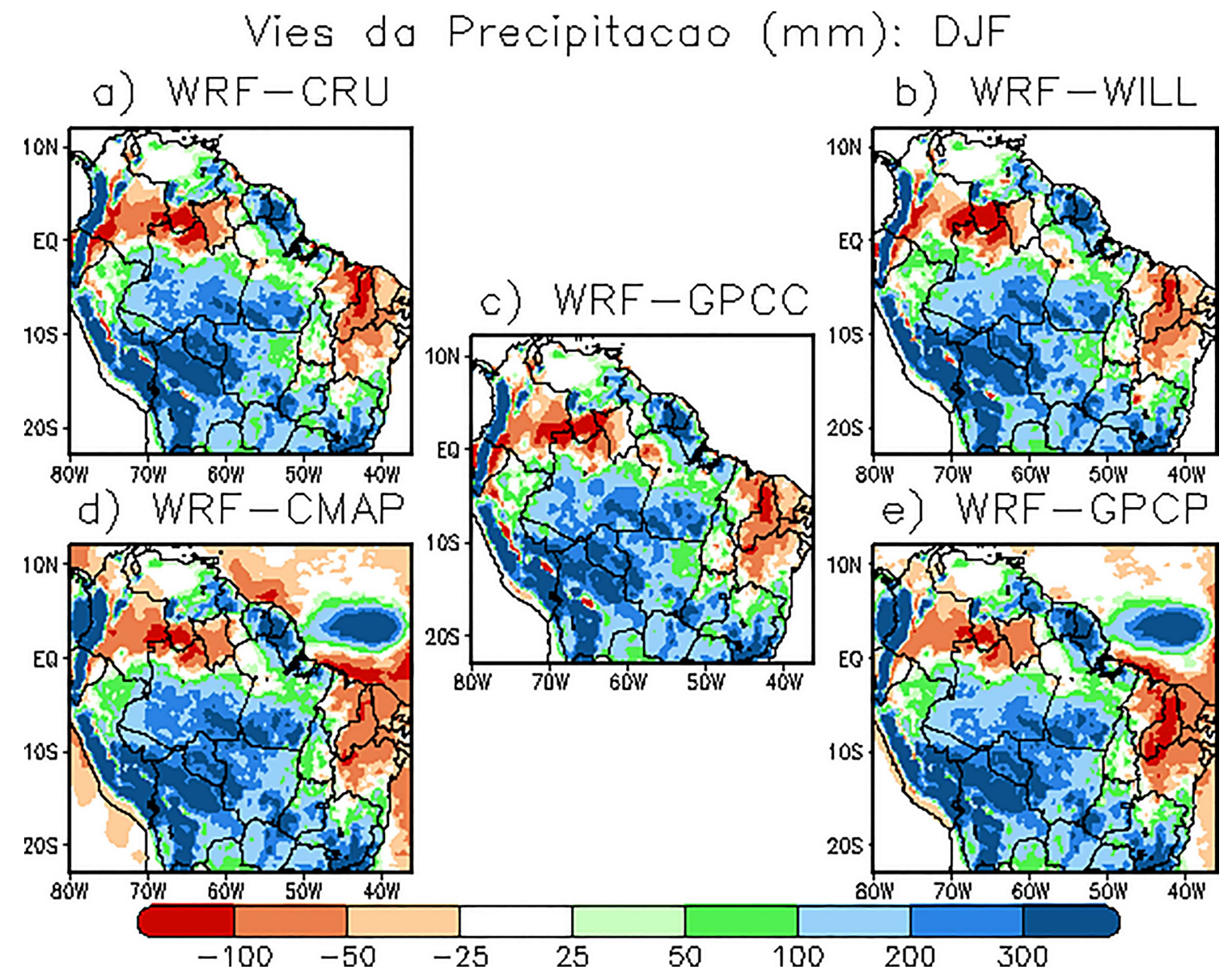

Figura 7 - Viés entre a precipitação simulada pelo WRF e as bases observadas: (a) WRF-CRU, (b) WRF-WILL, (c) WRF-GPCC, (d) WRF-CMAP e (e) WRF-GPCP; durante o trimestre DJF, de 1988 a 1999, na escala de $45 \mathrm{~km}$.

no setor central da Amazônia as áreas de maior correlação são verificadas mais consistentemente em relação aos dados CMAP (Fig. 9d) e GPCP (Fig. 9e). Essas diferenças decorrem dos erros de amostragem das observações de pluviômetros, e ao fato das estimativas de precipitação por satélites sobre o continente conter fontes de erros aleatórios, devido à natureza indireta da relação entre a cobertura de nuvens e a precipitação associada, além das imperfeições do algoritmo (Xie e Arkin, 1997).

Estes resultados têm implicações para o uso de WRF no NEB, porque a estação chuvosa nesta região concentrase no trimestre MAM, período em que o WRF tem menor subestimativa da precipitação e alta correlação em toda essa região, em relação a todas as bases de dados (Figs. 10a-10e). De maneira semelhante, altas correlações são encontradas principalmente nas áreas da Amazônia Oriental e ao longo da região de influência da ZCIT durante MAM nos dados CMAP (Fig. 10d) e GPCP (Fig. 10e), o que confirma a habilidade do modelo WRF na representação da precipitação e o seu potencial de previsibilidade nestas áreas. Em geral, o modelo apresenta habilidade para representar as principais características da distribuição da precipitação climatológica sobre a Amazônia, sem o viés seco, típico dos modelos de circulação global e outros modelos regionais apontados em outros estudos (Cavalcanti et al., 2002; Pesquero et al., 2010).

\subsubsection{Viés e correlação na escala de $15 \mathrm{~km}$}

Nas Figs. 11a-11b são apresentados os mapas de viés para o domínio D02 determinado a partir dos dados RCPH, nas estações de DJF (Fig. 11a) e MAM (Fig. 11b). Viés negativo predomina sobre regiões com baixa topografia e ao longo do rio Amazonas, tanto em DJF como em MAM, onde se verifica menor potencial de convecção, favorecendo menor precipitação conforme os estudos observacionais nesta região (Oliveira e Fitzjarrald, 1993; Fitzjarrald et al., 2008). Valores positivos de viés ocorrem no restante do domínio, mas com máximos valores ocorrendo ao longo da região costeira do Amapá e no extremo oeste do domínio D02, em DJF (Fig. 11a). As áreas com viés positivo se expandem para o setor nordeste do Pará em MAM (Fig. 11b).

Os mapas de correlação são apresentados nas Figs. 12a-12b, onde se verifica a ocorrência de áreas mais 


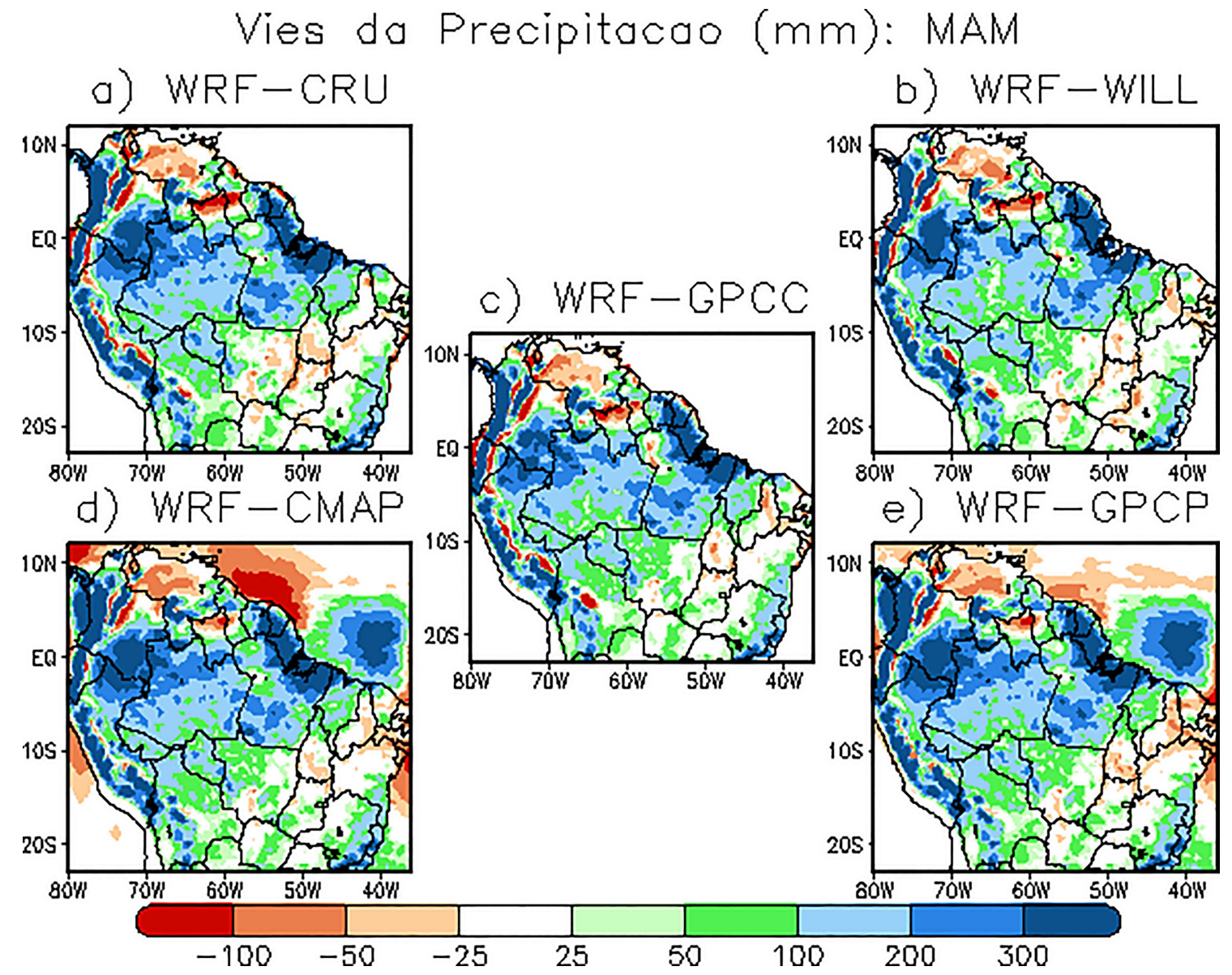

Figura 8 - Viés entre a precipitação simulada pelo WRF e as bases observadas: (a) WRF-CRU, (b) WRF-WILL, (c) WRF-GPCC, (d) WRF-CMAP e (e) WRF-GPCP; durante o trimestre MAM, de 1988 a 1999, na escala de $45 \mathrm{~km}$.

abrangentes com potencial previsibilidade da precipitação em DJF (valores de alta correlação) no oeste do Pará e leste do Amazonas (Fig. 12a). As áreas de maior correlação se expandem de maneira aleatória em MAM (Fig. 12b), na comparação com DJF. Essa característica espacial dos mapas de correlação mostra grande variabilidade entre as estações, refletindo a complexidade de processos envolvidos na representação da precipitação nessa região, em que de sistemas meteorológicos de mesoescala interagindo com a circulação de grande escala, tais como a proximidade da ZCIT e o mecanismo de circulação de brisa, estabelecem condição para formação de linhas de instabilidade e complexos convectivos que se formam no litoral ou em áreas continentais nessa época do ano (Cohen et al., 1995).

Considerando a área comum dos domínios D02 e D01, os mapas de viés para a escala de $15 \mathrm{~km}$ mostram valores de menores magnitudes em ambas as estações (DJF e MAM) do que os mapas de viés para a escala de $45 \mathrm{~km}$. Nesta mesma área comum, os mapas de correlações mostram áreas com valores maiores na escala de $15 \mathrm{~km}$ do que na escala de $45 \mathrm{~km}$, em DJF (Fig. 12a) e
MAM (Fig. 12b). Isso é verificado quando se comparam os mapas de correlação das simulações de $45 \mathrm{~km}$ com os dados CMAP (Fig. 9d) e GPCP (Fig. 9e), com os mapas de correlações da escala de $15 \mathrm{~km}$, com a RCPH (Figs. 12a-12b). Esta análise enfatiza a importância de conjuntos de dados de observação mais refinados, tais como a base RPCH, para avaliação de desempenho dos modelos climáticos aplicados em alta resolução espacial. Pois, ao mesmo tempo em que, o uso de várias escalas adiciona o potencial aumento da previsibilidade da precipitação, em especial na Amazônia, os processos associados em escala adequada, são capturados e representados pelo modelo na aplicação da técnica de refinamento de escalas.

\subsubsection{Análise geral dos índices de desempenho}

As Tabelas 3 e 4 mostram o viés médio calculado nas Caixas 01-08 no domínio D01 (45 km) comparado às observações globais de precipitação, e nas Caixas 04-06 para a grade refinada $(15 \mathrm{~km})$ considerando a base de dados RCPH. Adicionalmente é apresentado o viés calculado considerando as precipitações simuladas nos pontos 


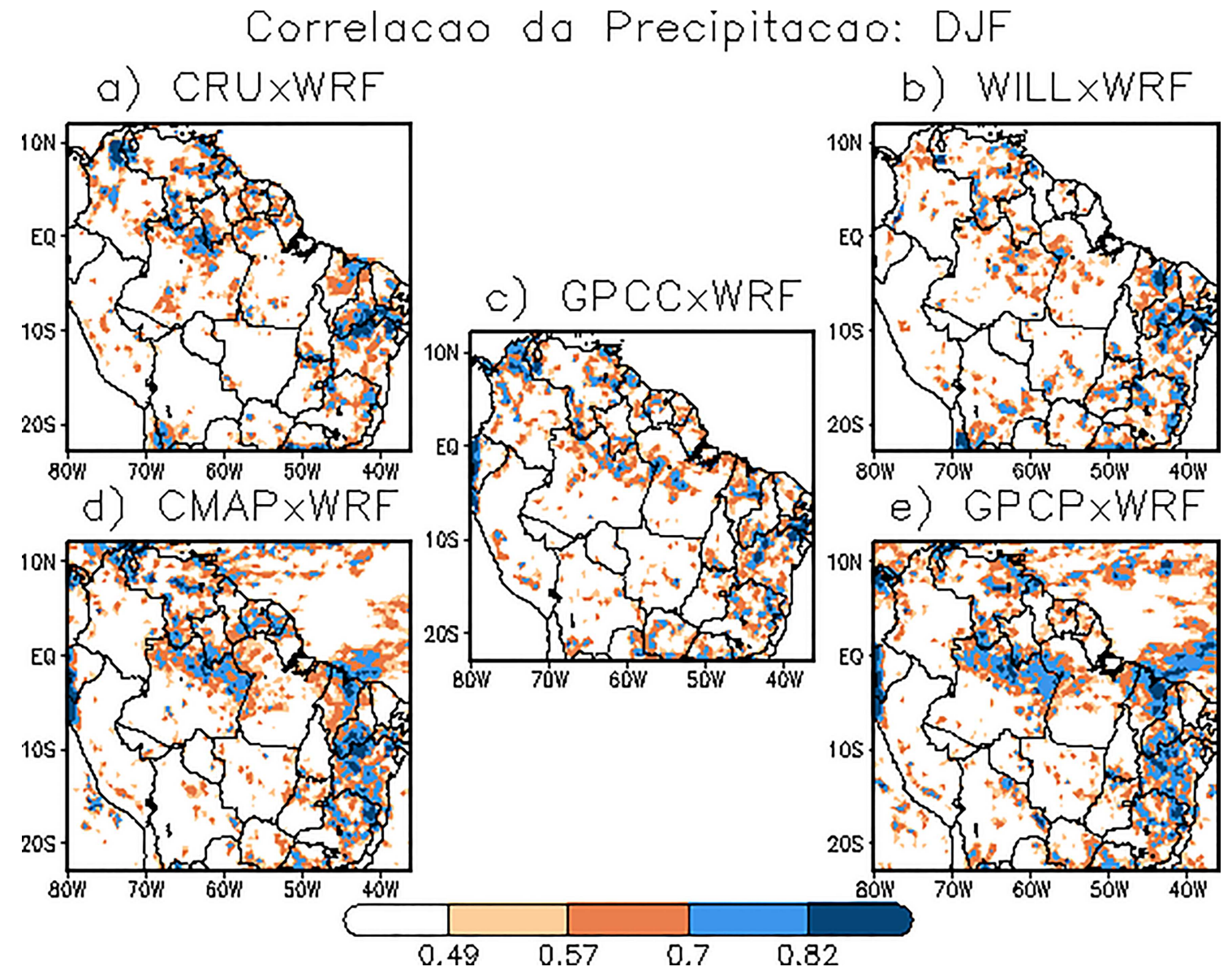

Figura 9 - Correlação entre a precipitação observada e simulada pelo WRF: (a) CRUxWRF, (b) WILLxWRF, (c) GPCCxWRF, (d) CMAPxWRF e (e) GPCPxWRF; durante o trimestre DJF, de 1988 a 1999, na escala de $45 \mathrm{~km}$.

de grid history e as medidas nas estações meteorológicas (escala local) do INMET, listadas na Tabela 2. A comparação utiliza a média dos pontos de estações dentro de cada caixa tanto para as observações quantos para as simulações. Neste caso, o objetivo foi de comparar as precipitações simuladas e observadas sem o efeito de interpolação das bases de dados em grade. A notável variação dos valores do viés entre as caixas indica uma dependência regional na habilidade do modelo para representar a precipitação e, ao mesmo tempo, mostra que essa dependência é também fruto de falta de representatividades de características locais nas bases de dados observacionais.

Tabela 3 - Viés médio da Precipitação (mm) em relação aos dados observados, estações INMET e RPCH durante DJF para período de 1988 a 1999, indicando os domínios e as Caixas.

\begin{tabular}{ccccccccc}
\hline \multicolumn{10}{c}{ VIÉS - DJF } \\
\hline & CMAP & CRU & GPCC & GPCP & WILL & INMET & RPCH \\
\hline Cx01 & 45 & 34 & 14 & 22 & 41 & 26 & - & - \\
Cx02 & 45 & -80 & -57 & -76 & -79 & -83 & 46 & - \\
Cx03 & 45 & 166 & 155 & 139 & 147 & 117 & 229 & - \\
\hline
\end{tabular}

Para a grade de $45 \mathrm{~km}$, o modelo apresenta uma predominância de viés positivo em ambas as estações do ano (Tabelas 3-4), considerando todos os conjuntos de dados

Tabela 4 - Viés médio da Precipitação (mm) em relação aos dados observados, estações INMET e RPCH durante MAM para período de 1988 a 1999, indicando os domínios e as Caixas.

\begin{tabular}{|c|c|c|c|c|c|c|c|c|}
\hline \multicolumn{9}{|c|}{ VIÉS - MAM } \\
\hline & & CMAP & CRU & GPCC & GPCP & WILL & INMET & $\mathrm{RPCH}$ \\
\hline $\mathrm{Cx} 01$ & 45 & 38 & -9 & 5 & 40 & 5 & - & - \\
\hline $\mathrm{Cx} 02$ & 45 & 267 & 253 & 217 & 266 & 199 & 334 & - \\
\hline $\mathrm{Cx} 03$ & 45 & 209 & 185 & 151 & 205 & 119 & 234 & - \\
\hline \multirow[t]{2}{*}{$\mathrm{Cx} 04$} & 45 & 185 & 178 & 167 & 182 & 448 & 170 & - \\
\hline & 15 & 134 & 128 & 117 & 131 & 102 & -195 & - \\
\hline \multirow[t]{2}{*}{$\mathrm{Cx} 05$} & 45 & 158 & 137 & 136 & 161 & 422 & 99 & 132 \\
\hline & 15 & 92 & 71 & 70 & 96 & 57 & -236 & 67 \\
\hline \multirow[t]{2}{*}{$\mathrm{Cx} 06$} & 45 & 317 & 395 & 254 & 305 & 621 & 301 & 161 \\
\hline & 15 & 296 & 374 & 233 & 284 & 241 & -329 & 140 \\
\hline $\mathrm{Cx} 07$ & 45 & 174 & 102 & 112 & 174 & 126 & 112 & - \\
\hline $\mathrm{Cx} 08$ & 45 & 133 & 127 & 115 & 132 & 110 & - & - \\
\hline
\end{tabular}




\section{Correlacao da Precipitacao: MAM}

a) CRUXWRF
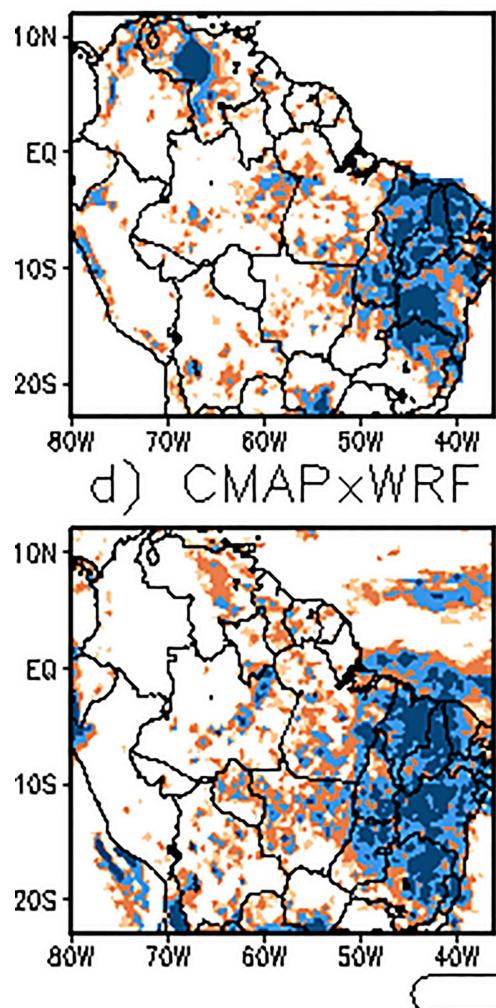

10
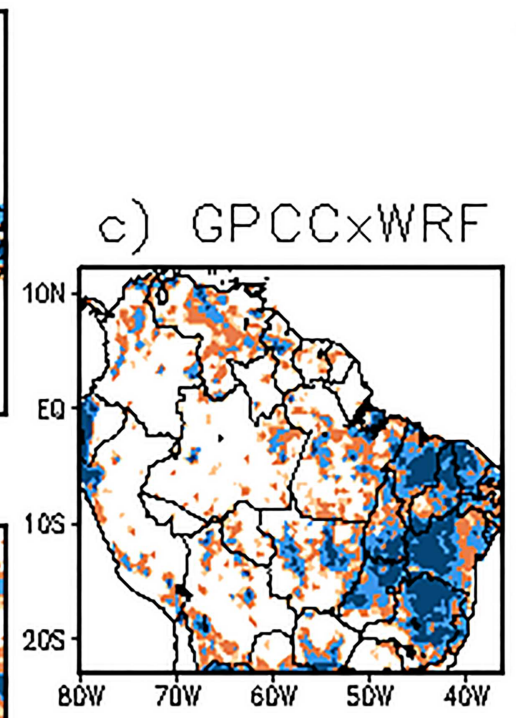

b) WILLXWRF

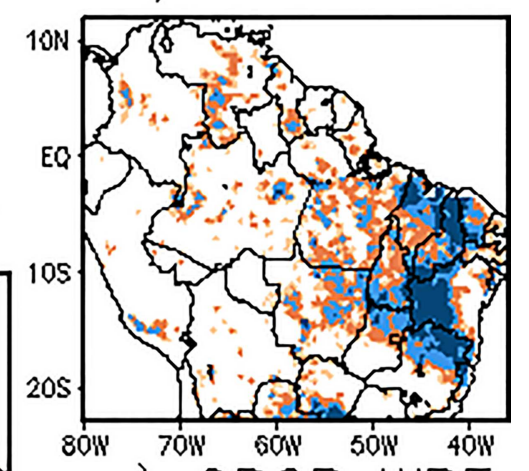

e) GPCPXWRF

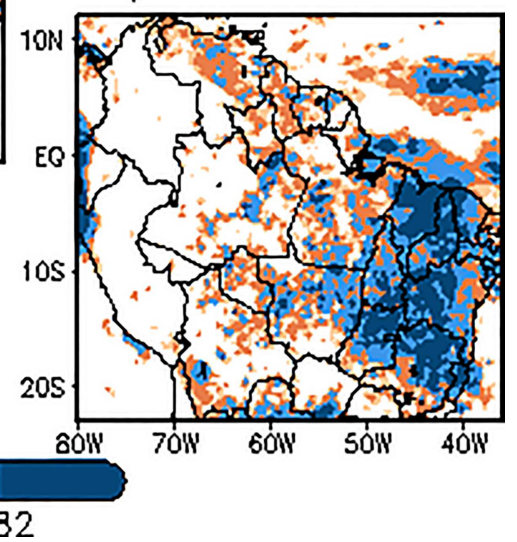

Figura 10 - Correlação entre a precipitação observada e simulada pelo WRF: (a) CRUxWRF, (b) WILLxWRF, (c) GPCCxWRF, (d) CMAPxWRF e (e) GPCPxWRF; durante o trimestre MAM, de 1988 a 1999, na escala de $45 \mathrm{~km}$.

de precipitação em grade, RCPH e do INMET. Exceção a isso é a Caixa 02 durante DJF (Tabela 3), com viés negativo em relação aos dados observados (CMAP, GPCP, CRU, WILL e GPCC), e viés positivo em relação aos dados do INMET. Nas Caixas 04 e 06, em que os resultados do domínio de $15 \mathrm{~km}$ são também avaliados, o modelo tem predominância de viés positivo em ambas as estações, porém com valores menores aos verificados na escala de $45 \mathrm{~km}$ (Tabelas 3-4), com exceção aos dados INMET em que o viés é negativo. A exceção é a Caixa 05 , em DJF, com viés negativo em relação aos dados CMAP, CRU, GPCC, GPCP, WILL e INMET (Tabela 3).

Quando as simulações são comparadas aos dados globais de precipitação, o modelo na grade de $45 \mathrm{~km}$ tem uma boa habilidade para representar a precipitação no extremo norte (Caixa 01) e no leste (Caixa 05) da Amazônia brasileira, durante as duas estações. A exceção ocorre em relação ao CRU em MAM, com viés de $-9 \mathrm{~mm}$. Em relação à base do INMET, os menores vieses são observados em DJF na Caixa 06 (superestimativa de $19 \mathrm{~mm}$ ) e Caixa 05 (subestimativa de $-1 \mathrm{~mm}$ ). Considerando todos os conjuntos de dados observados de precipitação e do INMET, em $45 \mathrm{~km}$, o viés durante o trimestre MAM (Tabela 4), nas Caixas 02-07, são positivos e maiores do que os correspondentes vieses na estação DJF (Tabela 3).

A estimativa do viés na escala de $15 \mathrm{~km}$, e seu comportamento frente à escala de $45 \mathrm{~km}$, é analisada considerando as Caixas 04-06 (Tabelas 3-4). Na Caixa 04, os valores dos vieses são positivos e menores na escala de $15 \mathrm{~km}$, em DJF (Tabela 3) e MAM (Tabela 4), para todas as bases de dados observacionais em grade, com exceção dos dados INMET em que o viés é negativo. Na Caixa 05, predomina o viés baixo e negativo em relação as bases de dados CMAP, CRU, GPCP e WIL, enquanto é negativo e alto para INMET, predominantemente no trimestre DJF (Tabela 3). Em MAM os vieses são positivos e maiores nas Caixas 04 e 05 , porém, com valores menores ao da escala de $45 \mathrm{~km}$ (Tabela 4). A proximidade da Caixa 06 à costa leste, favoreceu o aumento do viés na escala de $15 \mathrm{~km}$ em relação a de $45 \mathrm{~km}$, resultado do favorecimento a convergência de umidade e maior sensibilidade das físicas de convecção e microfísica de nuvem à uma maior disponibilidade de energia convectiva. 
Vies da Precipitacao $(\mathrm{mm}): \operatorname{WRF}(15 \mathrm{~km})-\mathrm{RPCH}$

a) D.JF

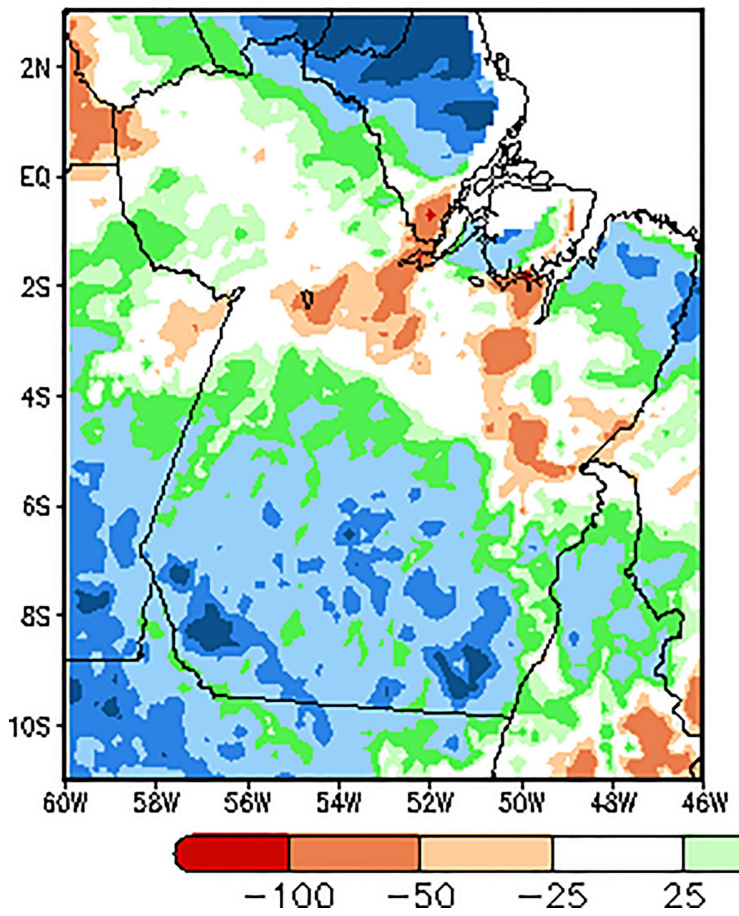

b) MAM

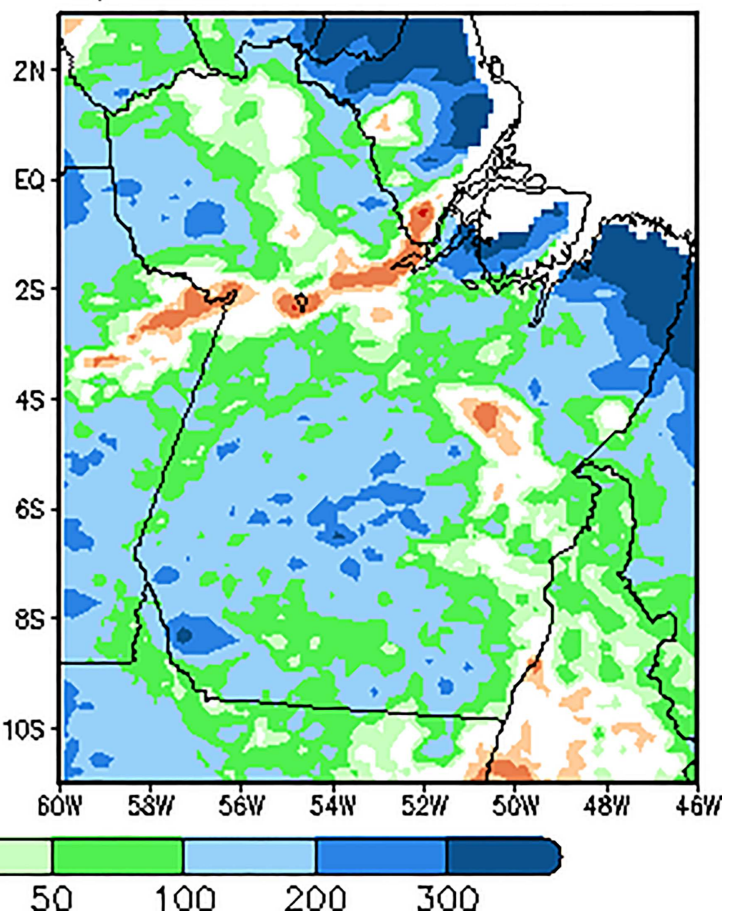

Figura 11 - Viés entre as precipitações simuladas (WRF) - na escala de $15 \mathrm{~km}$-,e observada (RPCH) durante os trimestres (a) DJF e (b) MAM, no período de 1988 a 1999

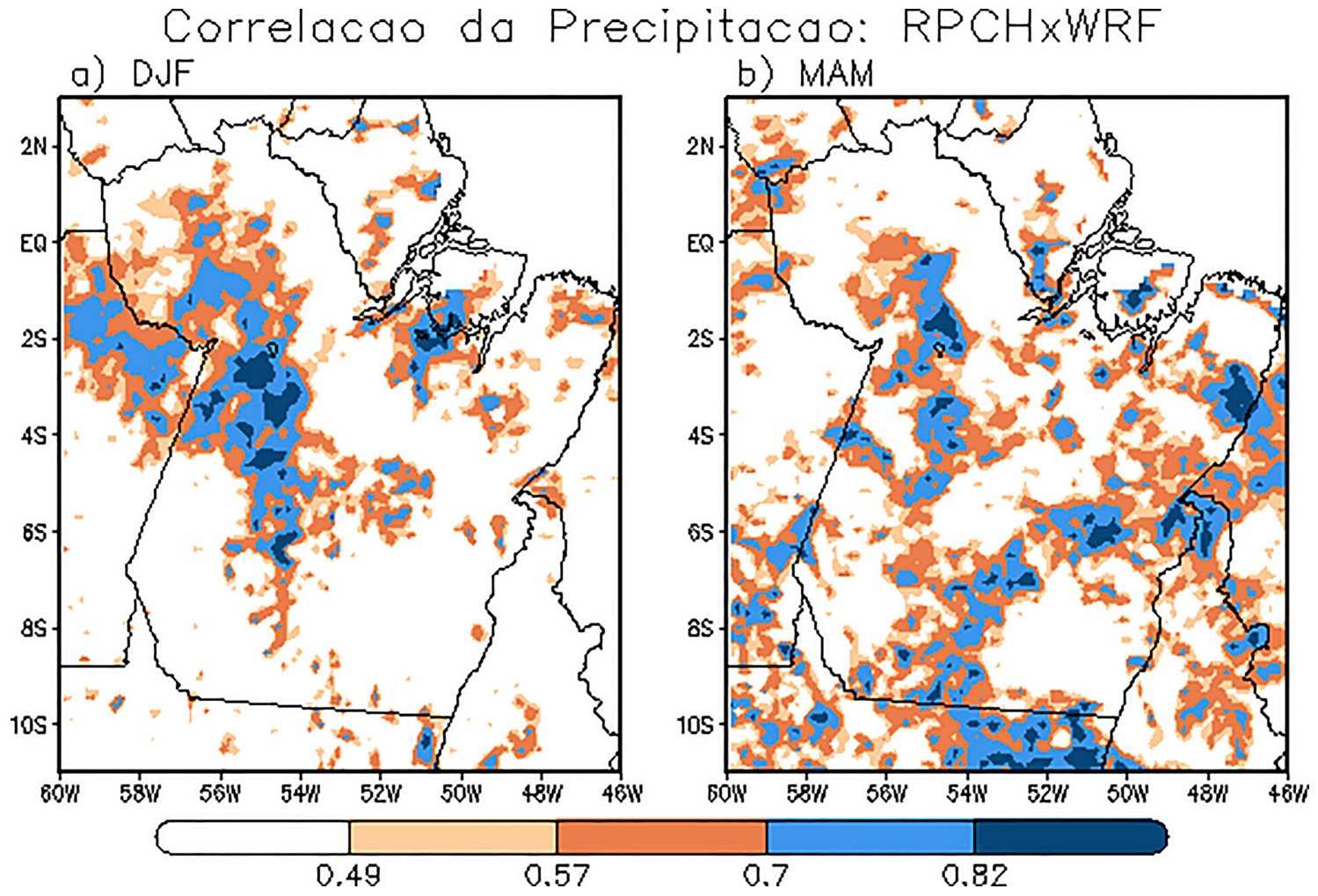

Figura 12 - Correlação entre as precipitações simuladas (WRF) - na escala de 15 km - e observada (RPCH) durante os trimestres (a) DJF e (b) MAM, no período de 1988 a 1999. 
A análise adicional para a escala de $15 \mathrm{~km}$ utilizando a base de dados RCPH nas Caixas 5-6, mostra que, nesta escala, o viés é também positivo nas estações DJF e MAM (Tabelas 3-4) porém, seu valor é bastante reduzido, principalmente na estação de MAM. O viés calculado usando a base de dados RCPH, quando comparado à simulação na escala de $45 \mathrm{~km}$, também apresenta redução (Tabelas 3-4). Nas Caixas 04-06 para os dados do INMET, ocorrem vieses, respectivamente, de $-195,-236$ e $-329 \mathrm{~mm}$. Isso mostra que os erros sistemáticos são também inerentes aos dados observacionais, que dificulta a sua comparação em escala compatível a da simulação do modelo.

Nas Tabelas 5-6 são apresentadas as análises da REQM para as simulações nos domínios D01, D02 e para os pontos de estações do INMET, que refletem resultados similares aos do viés para as todas as áreas das sub-regiões (Tabelas 3-4). Considerando as simulações com a grade de

Tabela 5 - REQM da Precipitação (mm) em relação aos dados observados, estações INMET e RPCH durante DJF.

\begin{tabular}{|c|c|c|c|c|c|c|c|c|}
\hline \multicolumn{9}{|c|}{ REQM - DJF } \\
\hline & & CMAP & CRU & GPCC & GPCP & WILL & INMET & $\mathrm{RPCH}$ \\
\hline $\mathrm{Cx} 01$ & 45 & 64 & 56 & 53 & 70 & 64 & - & - \\
\hline $\mathrm{Cx} 02$ & 45 & 113 & 108 & 102 & 111 & 119 & 105 & - \\
\hline $\mathrm{Cx} 03$ & 45 & 195 & 179 & 167 & 174 & 144 & 256 & - \\
\hline \multirow[t]{2}{*}{ Cx04 } & 45 & 162 & 140 & 156 & 145 & 152 & 178 & - \\
\hline & 15 & 149 & 126 & 143 & 132 & 137 & 208 & - \\
\hline \multirow[t]{2}{*}{ Cx05 } & 45 & 111 & 109 & 107 & 111 & 108 & 88 & 109 \\
\hline & 15 & 86 & 91 & 78 & 86 & 89 & 240 & 79 \\
\hline \multirow[t]{2}{*}{ Cx06 } & 45 & 165 & 202 & 135 & 156 & 162 & 130 & 131 \\
\hline & 15 & 179 & 225 & 131 & 170 & 163 & 322 & 113 \\
\hline Cx07 & 45 & 311 & 222 & 240 & 305 & 254 & 168 & - \\
\hline Cx08 & 45 & 299 & 284 & 263 & 306 & 266 & - & - \\
\hline
\end{tabular}

Tabela 6 - REQM da Precipitação (mm) em relação aos dados observados, estações INMET e RPCH durante MAM.

\begin{tabular}{|c|c|c|c|c|c|c|c|c|}
\hline \multicolumn{9}{|c|}{ REQM - MAM } \\
\hline & & CMAP & CRU & GPCC & GPCP & WILL & INMET & $\mathrm{RPCH}$ \\
\hline $\mathrm{Cx} 01$ & 45 & 86 & 81 & 67 & 84 & 65 & - & - \\
\hline Cx02 & 45 & 331 & 308 & 274 & 333 & 258 & 366 & - \\
\hline $\mathrm{Cx} 03$ & 45 & 220 & 200 & 162 & 217 & 134 & 249 & - \\
\hline \multirow[t]{2}{*}{$\mathrm{Cx} 04$} & 45 & 202 & 202 & 186 & 197 & 178 & 189 & - \\
\hline & 15 & 151 & 153 & 136 & 146 & 128 & 196 & - \\
\hline \multirow[t]{2}{*}{$\mathrm{Cx} 05$} & 45 & 191 & 182 & 168 & 193 & 164 & 195 & 165 \\
\hline & 15 & 131 & 128 & 106 & 128 & 115 & 247 & 103 \\
\hline \multirow[t]{2}{*}{$\mathrm{Cx} 06$} & 45 & 353 & 428 & 299 & 343 & 312 & 390 & 241 \\
\hline & 15 & 313 & 392 & 255 & 302 & 265 & 343 & 191 \\
\hline Cx07 & 45 & 200 & 145 & 141 & 200 & 154 & 149 & - \\
\hline Cx08 & 45 & 176 & 170 & 155 & 175 & 156 & - & - \\
\hline
\end{tabular}

$45 \mathrm{~km}$ e os conjuntos de dados globais, os valores mais baixos de REQM em DJF ocorrem na Caixa 01 seguido pelas Caixa 02 e Caixa 05 (Tabela 5) e, em MAM, na Caixa 01, seguido pelas Caixa 04 e Caixa 05 (Tabela 6). A REQM para a grade aninhada $(15 \mathrm{~km})$ tem valores menores do que os correspondentes do domínio principal $(45 \mathrm{~km})$, nas Caixa 04 e Caixa 05 para ambos os trimestres (DJF e MAM). Em especial, na Caixa 06 (em MAM), há redução dos valores da REQM e, em DJF ocorre o contrário, considerando todos os conjuntos de dados globais e INMET. De maneira semelhante à análise do viés, o uso da base de dados RCPH na avaliação do REQM mostra uma redução do erro sistemático da precipitação nas Caixa 05 e Caixa 06, em ambas as estações, reforçando que os erros são também inerentes as observações e dependentes das configurações físicas do modelo. O principal resultado obtido aqui é a clara tendência de redução dos erros sistemáticos do modelo quando se combina refinamento de escalas, esquemas físicos que incorporem os mecanismos necessários a representação dos processos convectivos em suas diferentes escalas e, a disponibilidade de dados observacionais com maior densidade da rede de monitoramento.

\section{Conclusão}

O presente estudo avaliou o desempenho do modelo regional WRF em simular a precipitação durante a estação chuvosa da região Amazônica usando domínios aninhados (45 e $15 \mathrm{~km}$ ) em uma estratégia de downscaling dinâmico, e verificou-se que, o modelo foi capaz de representar a precipitação associada à ZCIT e ZCAS, que são os principais sistemas que modulam a precipitação durante a estação chuvosa na Amazônia mas, no entanto, a ZCAS é simulada ligeiramente ao sul de sua posição climatológica observada. Outro resultado importante é que a redução de escala melhora a distribuição espacial da precipitação na maior parte da região de estudo, o que confirma que, as características da superfície e sua influência nos sistemas locais responsáveis pela precipitação, foram contempladas no refinamento de escala. O modelo apresenta erros sistemáticos na simulação da precipitação que dependem da região, da escala, da estação do ano e, da representatividade das observações. Considerando a escala de $45 \mathrm{~km}$, os menores erros ocorreram nos setores norte e leste da Amazônia brasileira, erros intermediários, nas porções oeste e central, e os maiores na porção sul. Com uma grade mais refinada, os menores erros ocorreram na Amazônia central e os maiores, próximo à costa. Os resultados mostram que o refinamento de escala incorpora a influência das características locais, com diferentes contribuições entre o interior e zonas costeiras, na formação da precipitação. Comparando as simulações em uma escala de $15 \mathrm{~km}$ com os dados observados, o downscaling dinâmico confirma a tendência de melhor desempenho do modelo WRF em 
representar a precipitação em áreas continentais do Amazonas, onde há forte contraste para diferentes tipos de superfície tais como florestas e rios e, onde o efeito de brisa favorece a ocorrência de chuvas ao longo das margens. Assim, os resultados indicam que a técnica de downscaling dinâmico com o modelo WRF pode agregar valores à previsão climática sazonal para a região Amazônica e em estudos de impacto.

\section{Agradecimentos}

Este trabalho é parte da tese de doutorado do primeiro autor desenvolvido no Instituto Nacional de Pesquisas da Amazônia (INPA) e a Universidade Estadual do Amazonas (UEA), no período de 2009 a 2013, com apoio financeiro da Fundação de Amparo à Pesquisa do Estado do Amazonas (FAPEAM). Após este período, o mesmo foi apoiado pelo Programa de Apoio à Fixação de Doutores no Amazonas (FIXAM/FAPEAM - Edital nº 022/2013). Prestígios são devidos à FAPEAM (Processo: 062.00800/ 2015), CAPES (Processo: 88887.115874/2015-01), FINEP (REMCLAM-UEA) e CNPq (Processos 305942/ 2015-8 e 302679/2013-8 - parcialmente para o quarto e quinto autores, respectivamente). A todos, os nossos mais sinceros e profundos agradecimentos.

\section{Referências}

ADAMS, D.K.; SOUZA, E.P. DE; COSTA, A.A. Convecção úmida na Amazônia: implicações para modelagem numérica. Revista Brasileira de Meteorologia, v. 24, n. 2, p. 168-178, 2009.

ALVES, J.M.B.; BRISTOT, G.; COSTA, A.A.; MONCUNNIL, D.F.; SILVA, E.M.; et al. Uma aplicação da técnica de "downscaling" dinâmico no setor norte da região nordeste do Brasil. Revista Brasileira de Meteorologia, v. 18, n. 2, p. 161-180, 2003.

ALVES, J.M.B.; CAMPOS, J.N.B.; NASCIMENTO, L.S.V. Sensibilidade intrasazonal de um downscaling dinâmico de precipitação (1971-2000): Uma análise na bacia hidrográfica do Açude Castanhão-CE. Revista Brasileira de Meteorologia, v. 23, n. 1, p. 73-87, 2008.

ALVES, L.M.; MARENGO, J.A. Assessment of regional seasonal predictability using the PRECIS regional climate modeling system over South America. Theoretical and Applied Climatology, v. 100, n. 3-4, p. 337-350, 2009.

CARVALHO, D.; ROCHA, A.; GÓMEZ-GESTEIRA, M.; SANTOS, C. A sensitivity study of the WRF model in wind simulation for an area of high wind energy. Environmental Modelling and Software, v. 33, p. 23-34, 2012.

CAVALCANTI, I.F.A.; MARENGO, J.A.; SATYAMURTY, P.; NOBRE, C.A.; TROSNIKOV, I.; et al. Global climatological features in a simulation using the CPTEC-COLA AGCM. Journal of Climate, v. 15, n. 21, p. 2965-2988, 2002.
CHOU, S.C.; MARENGO, J.A.; LYRA, A.A.; SUEIRO, G.; PESQUERO, J.F.; et al. Downscaling of South America present climate driven by 4-member HadCM3 runs. Climate Dynamics, v. 38, n. 3-4, p. 635-653, 2012.

COHEN, J.C.P.; SILVA DIAS, M.A.F.; NOBRE, C.A. Aspectos climatologicos das linhas de instabilidade na Amazonia. Climanálise, v. 4, 34-40. 1989.

CORREAA, P.B.; CANDIDO, L.A.; SOUZA, R.A.F.; ANDREOLI, R.V.; KAYANO, M.T. Estudo do fenômeno da ilha de calor na cidade de Manaus/AM: Um estudo a partir de dados de sensoriamento remoto, modelagem e estações meteorológicas. Revista Brasileira de Meteorologia, v. 31, n. 2, p. 167-176, 2016.

DAI, A. Precipitation characteristics in eighteen coupled climate models. Journal of Climate, v. 19, n. 18, p. 4605-4630, 2006.

DANTAS, V.A.; AMORIM, A.C.B.; COSTA, M.S.; SILVA, C. M.S. Downscaling dinâmico sobre o Nordeste do Brasil utilizando um modelo climático regional: impacto de diferentes parametrizações na precipitação simulada. Revista Brasileira de Geografia Física, v. 6, n. 4, p. 995-1008, 2013.

FERREIRA, A.P.; CASTANHEIRA, J.M.; ROCHA, A.; FERREIRA, J. Estudo de sensibilidade das previsões de superfície em Portugal pelo WRF face à variação das parametrizações físicas. XXX Jornadas Científicas de la Associación Meteorológica Española, n. 1, p. 1-27, 2008.

FISCH, G.; MARENGO, J.A.; NOBRE, C.A. Uma Revisão geral sobre o clima da Amazônia. Acta Amazonica, v. 28, n. 2, p. 101-126, 1998.

FITZJARRALD, D.R.; SAKAI, R.K.; MORAES, O.L.L.; OLIVEIRA, R.C.; ACEVEDO, O.C.; et al. Spatial and temporal rainfall variability near the Amazon-Tapajós confluence. Journal of Geophysical Research, v. 113, n. G00B11, p. 1-17, 2008.

GIORGI, F. Simulation of regional climate using a limited area model nested in a general circulation model. Journal of Climate, v. 3, n. 9, p. 941-963, 1990.

GILLILAND, E.K.; ROWE, C.M. A comparison of cumulus parameterization schemes in the WRF model. In: Proceedings of the 87th AMS Annual Meeting \& 21th Conference on Hydrology. 2007.

HARRIS, L.M.; DURRAN, D.R. An idealized comparison of one-way and two-way grid nesting. Monthly Weather Review, v. 138, n. 6, p. 2174-2187, 2010.

HUFFMAN, G.J.; ADLER, R.F.; MORRISSEY, M.M.; BOLVIN, D.T.; CURTIS, S.; et al. Global precipitation at onedegree daily resolution from multisatellite observations. Journal of Hydrometeorology, v. 2, n. 1, p. 36-50, 2001.

KAIN, J.S.; FRITSCH, J.M. Convective parameterization for mesoscale models: The Kain-Fritsch scheme. American Meteorological Society, v. 24, n. 46, p. 165-170, 1993.

KAIN, J.S.; FRITSCH, J.M. A One-dimensional entraining/ detraining plume model and its application in convective parameterization. Journal of the Atmospheric Sciences, v. 47, n. 23, p. 2784-2802, 1990.

KINTER III, J.L.; PAOLINO, D.; STRAUS, D.; KIRTMAN, B.; MIN, D. Seasonal prediction with CCSM: Impact of atmosphere and land surface initialization, in: Science and Technology Infusion Climate Bulletin - Test Bed Joint 
Seminar Series, US National Oceanic and Atmospheric Administration Climate, NCEP, Camp Springs, Maryland, 4 May 2009.

KODAMA, Y. Large-scale common features of subtropical precipitation zones (the Baiu Frontal Zone, the SPCZ, and the SACZ) Part I: Characteristics of subtropical frontal zones. Journal of the Meteorological Society of Japan, v. 70, n. 4, p. 813-836, 1992.

LAPRISE, R.; KORNIC, D.; RAPAIC, M.; SEPAROVIC, L.; LEDUC, M.; et al. Considerations of domain size and large-scale driving for nested regional climate models: Impact on internal variability and ability at developing small-scale details, in: Climate Change, edited by: Berger, A., Mesinger, F., and Sijacki, D., Springer, Vienna, doi: 10.1007/978-3-7091-0973-1_14, p. 181-199, 2012.

LEIVAS, J.F.; RIBEIRO, G.G.; SARAIVA, I.; SANTO, J.S.E.; SOUZA, M.B.; et al. Avaliação dos prognósticos de precipitação simulada pelo modelo BRAMS na Amazônia Ocidental na estação chuvosa. Acta Amazonica, v. 41, n. 3, p. 347-354, 2011.

LEUNG, L.R.; KUO, Y.H.; TRIBBIA, J. Research needs and directions of regional climate modeling using WRF and CCSM. Bulletin of the American Meteorological Society, v. 87, n. 12, p. 1747-1751, 2006.

LIN, Y.-L.; FARLEY, R.D.; ORVILLE, H.D. Bulk parameterization of the snow field in a cloud model. Journal of Climate and Applied Meteorology, v. 22, n. 6, p. 1065-1092, 1983.

LITTA, A.J.; IDICULA, S.M.; MOHANTY, U.C. A comparative study of convective parameterization schemes in WRFNMM model. International Journal of Computer Applications, v. 33, n. 6, p. 32-39, 2011.

LIU, Y.; GIORGI, F.; WASHINGTON, W.M. Simulation of summer monsoon climate over east Asia with an NCAR regional climate model. Monthly Weather Review, v. 122, n. 10, p. 2331-2348, 1994.

MASS, C.F.; OVENS, D.; WESTRICK, K.; COLLE, B.A. Does increasing horizontal resolution produce more skillful forecasts? The results of two years of real-time numerical weather prediction over the Pacific Northwest. Bulletin of the American Meteorological Society, v. 83, n. 3, p. 407430, 2002.

MCCAA, J.R.; ROTHSTEIN, M.; EATON, B.E.; ROSINSKI, J. M.; KLUZEK, E.; et al. User's Guide to the NCAR Community Atmosphere Model (CAM 3.0), Climate And Global. p. 1-97, 2013.

MISRA, V.; DIRMEYER, P.A.; KIRTMAN, B.P. Dynamic downscaling of seasonal simulations over South America. Journal of Climate, v. 16, n. 1, p. 103-117, 2003.

MITCHELL, T.D.; JONES, P.D. An improved method of constructing a database of monthly climate observations and associated high-resolution grids. International Journal of Climatology, v. 25, n. 6, p. 693-712, 2005.

JUAREZ, R.I.N.; LI, W.; FU, R.; FERNANDES, K. Comparison of precipitation datasets over the tropical South American and African continents. Journal of Hydrometeorology, v. 10, n. 1, p. 289-299, 2009.

OLIVEIRA, E.M.G. Parametrizações convectivas no modelo WRF e sua relação com a precipitação durante ciclogêneses no sudeste da América do Sul. Dissertação de
Mestrado. Universidade Federal de Santa Maria, RS, p. 95, 2014.

OLIVEIRA, A.P.; FITZJARRALD, D.R. The Amazon river breeze and the local boundary layer: I. Observations. Boundary-Layer Meteorology, v. 63, n. 1, p. 141-162, 1993.

OYAMA, M.D.; ROCHA, E.J.P.; NOBRE, C.A. Estudo preliminar sobre o tempo de "spin up" da umidade do solo no modelo climático do CPTEC. In: XI Congresso Brasileiro de Meteorologia, p. 16-20, 2000.

PESQUERO, J.F.; CHOU, S.C.; NOBRE, C.A.; MARENGO, J. A. Climate downscaling over South America for 19611970 using the ETA model. Theoretical and Applied Climatology, v. 99, p. 75-93, 2010.

REBOITA, M.S.; GAN, M.A., ROCHA, R.P.; AMBRIZZI, T. Regimes de precipitação na América do Sul: uma revisão bibliográfica. Revista Brasileira de Meteorologia, v. 25, n. 2, p. 185-204, 2010.

REYNOLDS, R.W.; MARSICO, D.C. An Improved real-time global sea surface temperature analysis. Journal of Climate, v. 6, p. 114-119, 1993.

RIBEIRO, A.; VICTORIA, R.L; PEREIRA, A.R.; VILLA NOVA, N.A. MARTINELLI, L.A.; et al. Análise do regime pluviométrico da região Amazônica a partir de dados de onze localidades. Revista Brasileira de Meteorologia, v. 11, n. 1-2, p. 25-35, 1996.

ROCHA, R.P.; MORALES, C.A.; CUADRA, S.V.; AMBRIZZI, T. Precipitation diurnal cycle and summer climatology assessment over South America: An evaluation of regional climate model version 3 simulations. Journal of Geophysical Research, v. 114, n. D10, p. 1-19, 2009.

RUDOLF, B.; HAUSCHILD, H.; RUETH, W.; SCHNEIDER, U. Terrestrial precipitation analysis: operational method and required density of point measurements. Global Precipitations and Climate Change, v. NATO ASIS, p. 173186, 1994.

SAHA, S.; MOORTHI, S.; PAN, H.-L.; WU, X.; WANG, J.; et al. The NCEP climate forecast system reanalysis. Bulletin of the American Meteorological Society, v. 91, n. 8, p. 1015-1057, 2010.

SALES, D.C.; COSTA, A.A.; SILVA, E.M.; JÚNIOR, F.C.V.; CAVALCANTE, A.M.B.; et al. Projeções de mudanças na precipitação e temperatura no Nordeste Brasileiro utilizando a técnica de downscaling dinâmico. Revista Brasileira de Meteorologia, v. 30, n. 4, p. 435-456, 2015.

SAMPAIO, G.; DIAS, P.L.S. Evolução dos modelos climáticos e de previsão de tempo e clima. Revista USP, n. 103, p. 4154, 2014.

SHARMA, A.; HUANG, H.P. Regional climate simulation for Arizona: Impact of resolution on precipitation. Advances in Meteorology, v. 2012, p. 13, 2012.

SILVA, R.R.; DIAS, P.L.S.; MOREIRA, D.S.; SOUZA, E.B. Modelo OLAM (ocean-land-atmosphere-model): Descrição, aplicações, e perspectivas. Revista Brasileira de Meteorologia, v. 24, n. 2, p. 144-157, 2009.

SILVEIRA, C.S.; FILHO, F.A.S.; COSTA, A.A.; CABRAL, S.L. Avaliação de desempenho dos modelos do CMIP5 quanto à representação dos padrões de variação da precipitação no Século XX sobre a região Nordeste do Brasil, Amazônia e Bacia do Prata e análise das projeções para o Cenário 
RCP8.5. Revista Brasileira de Meteorologia, v. 28, n. 3, p. 317-330, 2013.

SILVESTRI, G.; VERA, C.; JACOB, D.; PFEIFER, S.; TEICHMANN, C. A high-resolution 43-year atmospheric hindcast for South America generated with the MPI regional model. Climate Dynamics, v. 32, n. 5, p. 693-709, 2009.

SKAMAROCK, W.C.; KLEMP, J.B.; DUDHIA, J.; GILL, D.O.; BARKER, D.M.; et al. A Description of the Advanced Research WRF version 3. Technical Report, p. 113, 2008.

SNYDER, M.A.; SLOAN, L.C. Transient future climate over the western United States using a regional climate model. Earth Interactions, v. 9, n. 11, p. 1-21, 2005.

SOLMAN, S.A. Regional climate modeling over South America: A review. Advances in Meteorology, v. 2013, p. 1-13, 2013.

SOUZA, E.B.; LOPES, M.N.G.; ROCHA, E.J.P.; SOUZA, J.R. S.; CUNHA, A.C.; et al. Precipitação sazonal sobre a Amazônia oriental no período chuvoso: Observações e simulações regionais com o RegCM3. Revista Brasileira de Meteorologia, v. 24, n. 2, p. 111-124, 2009.

SOUZA, E.B.; AMBRIZZI, T. Pentad precipitation climatology over Brazil and the associated atmospheric mechanisms. Climanálise, v. 2, n. 1972, p. 1-20, 2004.

SOUZA, E.P.; SILVA, E.M. Impacto da implementação de uma parametrização de convecção rasa em um modelo de mesoescala: Descrição e testes de sensibilidade do esquema. Revista Brasileira de Meteorologia, v. 18, n. 1, p. 33-42, 2003.
SUN, L.; MONCUNILL, D.F.; LI, H.; MOURA, A.D.; FILHO, F.A.S. Climate downscaling over Nordeste, Brazil, using the NCEP RSM97. Journal of Climate, v. 18, p. 551-567, 2005.

TRAIL, M.; TSIMPIDI, A.P.; LIU, P.; TSIGARIDIS, K.; HU,Y.; et al. Downscaling a global climate model to simulate climate change over the US and the implication on regional and urban air quality. Geoscientific Model Development, v. 6, n. 5, p. 1429-1445, 2013.

WILlMOTT, C.J.; ROBESON, S.M. Climatologically Aided Interpolation (CAI) of terrestrial air temperature. International Journal of Climatology, v. 15, n. 2, p. 221-229, 1995.

XIE, P.; JANOWIAK, J.E.; ARKIN, P.A.; ADLER, R.; GRUBER, A.; et al. GPCP pentad precipitation analyses: An experimental dataset based on gauge observations and satellite estimates. Journal of Climate, v. 16, n. 13, p. 2197-2214, 2003.

XIE, P.; ARKIN, P.A. Global precipitation: A 17-year monthly analysis based on gauge observations, satellite estimates, and numerical model outputs. Bulletin of the American Meteorological Society, v. 78, n. 11, p. 2539-2558, 1997.

This is an Open Access article distributed under the terms of the Creative Commons Attribution License, which permits unrestricted use, distribution, and reproduction in any medium, provided the original work is properly cited. 